PAPER • OPEN ACCESS

\title{
Inflationary magnetogenesis in the perturbative regime
}

To cite this article: Massimo Giovannini 2021 Class. Quantum Grav. 38135018

View the article online for updates and enhancements.

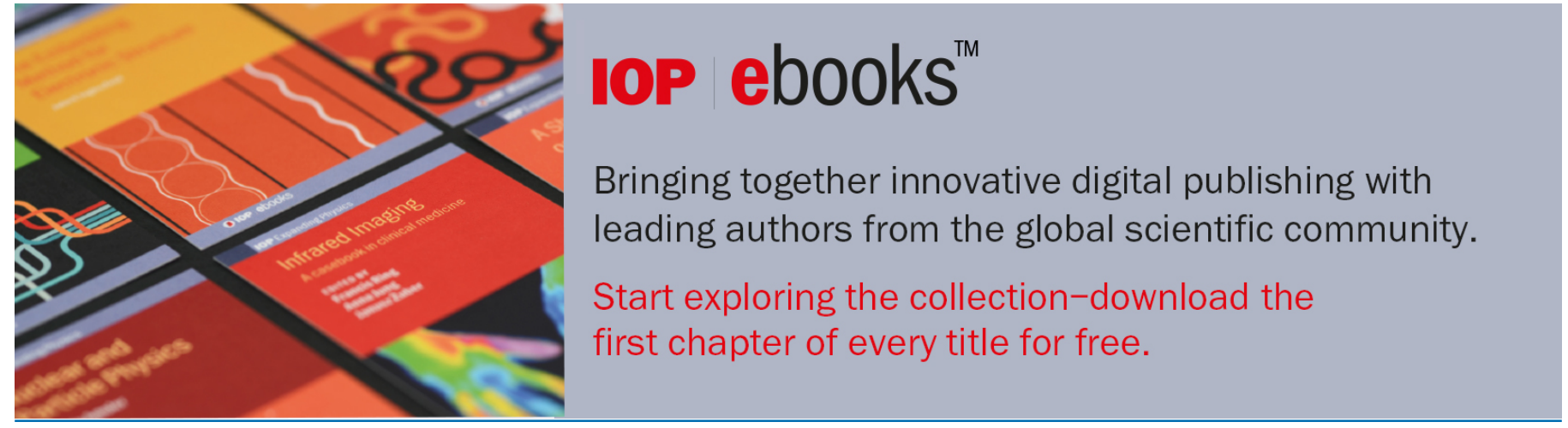

This content was downloaded from IP address 128.141 .192 .28 on 09/06/2021 at 08:48 


\title{
Inflationary magnetogenesis in the perturbative regime
}

\author{
Massimo Giovannini* \\ Department of Physics, CERN, 1211 Geneva 23, Switzerland \\ INFN, Section of Milan-Bicocca, 20126 Milan, Italy \\ E-mail: massimo.giovannini@cern.ch
}

Received 29 December 2020, revised 31 March 2021

Accepted for publication 15 April 2021

Published 3 June 2021

\begin{abstract}
While during inflation a phase of increasing gauge coupling allows for a scaleinvariant hyperelectric spectrum, when the coupling decreases a flat hypermagnetic spectrum can be generated for typical wavelengths larger than the effective horizon. After the gauge coupling flattens out the late-time hypermagnetic power spectra outside the horizon in the radiation epoch are determined by the hyperelectric fields at the end of inflation whereas the opposite is true in the case of decreasing coupling. Instead of imposing an abrupt freeze after inflation, we consider a smooth evolution of the mode functions by positing that the gauge couplings and their conformal time derivatives are always continuous together with the background extrinsic curvature. The amplified gauge power spectra are classified according to their transformation properties under the duality symmetry. After clarifying the role of the comoving and of the physical spectra in the formulation of the relevant magnetogenesis constraints, the parameter space of the scenario is scrutinized. It turns out that a slightly blue hyperelectric spectrum during inflation may lead to a quasi-flat hypermagnetic spectrum prior to matter radiation equality and before the relevant wavelengths reenter the effective horizon. In this framework the gauge coupling is always perturbative but the induced large-scale magnetic fields can be of the order of a few hundredths of a $\mathrm{nG}$ and over typical length scales between a fraction of the $\mathrm{Mpc}$ and $100 \mathrm{Mpc}$ prior to the gravitational collapse of the protogalaxy.
\end{abstract}

Keywords: cosmology, inflation, duality, magnetogenesis, scalar tensor theories of gravity, quantum field theory in curved backgrounds

(Some figures may appear in colour only in the online journal)

*Author to whom any correspondence should be addressed.

Original content from this work may be used under the terms of the Creative Commons Attribution 4.0 licence. Any further distribution of this work must maintain attribution to the author(s) and the title of the work, journal citation and DOI. 


\section{Introduction}

Besides the invariance under local gauge transformations, the Weyl [1] and the duality $[2,3]$ symmetries are particularly relevant for the dynamics of the gauge fields in general relativity and in scalar-tensor theories of gravity. If the governing equations of a given field are invariant under Weyl rescaling, the corresponding quantum fluctuations are not parametrically amplified by the evolution of the geometry [4-6]. In the absence of sources duality rotates field strengths into their duals (i.e. tensors into pseudotensors) and constrains the gauge power spectra potentially amplified during conventional or unconventional inflationary phases.

The symmetries of the evolution equations of the gauge fields in curved space-times are one of the main handles on the origin of the large-scale magnetism, a perplexing problem originally posed by Fermi [7] in connection with the propagation of cosmic rays within the Galaxy (see also [8]) and subsequently scrutinized by Hoyle [9] in a cosmological context in view of the comparatively large correlation scales of the fields. Indeed, while the typical diffusion scale in the interstellar medium is of the order of the $\mathrm{AU}(1 \mathrm{AU}=1.49 \times$ $10^{13} \mathrm{~cm}$ ) magnetic fields are observed over larger scales ranging between the $30 \mathrm{kpc}$ and few $\operatorname{Mpc}\left(1 \mathrm{pc}=3.08 \times 10^{18} \mathrm{~cm}\right)$.

Three general classes of suggestions have been proposed through the years. Soon after Hoyle's observations [9] Zeldovich [10] and of Thorne [11] suggested, in the context of anisotropic (but homogeneous) Bianchi-type models [12], that the large-scale magnetic fields could be a fossil remnant of a primordial field that originated with the Universe. In a complementary perspective we could imagine that the large-scale magnetic fields have been produced at some point during the radiation epoch and inside the Hubble radius by the vorticity associated with the turbulent dynamics; the first one suggesting this possibility was probably Harrison $[13,14]$ whose idea found direct applications in the context of phase transitions. Finally the third class of scenarios implies that a spectrum of gauge fields is produced because of the breaking of Weyl invariance during a standard stage of inflationary expansion; this is the perspective discussed in the present paper. The collection of themes related to the origin and to the early time effects of large-scale magnetism has been dubbed some time ago magnetogenesis [15]. While this terminology was so far quite successful, the problem itself has a long history as a number of inspiring monographs demonstrates [16-18]; see also references [19-21] for some more recent reviews.

It has been repeatedly argued through the years that magnetic fields with a sufficiently large correlation scale could be generated during a phase of accelerated expansion in full analogy with what happens for the scalar and tensor modes of the geometry (see e.g. [22]). The gauge fields parametrically amplified in the early Universe are in fact divergenceless vector random fields that do not break the spatial isotropy (as it happens instead in the case of the fossil remnants discussed in references $[10,11])$. To avoid the constraints imposed by Weyl invariance the gauge fields might directly couple to one or more scalar fields (see [23-26] for an incomplete list of references). The scalar fields may coincide with one or (more inflatons) or even with multiple spectator fields. This class of models is based on the following action:

$$
S_{\text {gauge }}=-\frac{1}{16 \pi} \int \mathrm{d}^{4} x \sqrt{-G}\left[\lambda(\varphi, \psi) Y_{\alpha \beta} Y^{\alpha \beta}+\lambda_{\text {pseudo }}(\varphi, \psi) Y_{\alpha \beta} \widetilde{Y}^{\alpha \beta}\right] .
$$

Within the present notations $Y^{\mu \nu}$ and $\widetilde{Y}^{\mu \nu}$ are, respectively, the gauge field strength and its dual; $G=\operatorname{det} G_{\mu \nu}$ is the determinant of the four-dimensional metric with signature mostly 
minus $^{1}$. In equation (1.1) $\varphi$ denotes the inflaton field while $\psi$ represents a generic spectator field. During a stage of conventional (slow-roll) inflation the variation of $\lambda$ is associated with the variation of the gauge coupling whose evolution may reach into the strong coupling regime (see the third paper of reference [23]). The presence of $\lambda(\varphi, \psi)$ in equation (1.1) is more relevant than the pseudoscalar (axion-like [27, 28]) coupling which will be ignored even if it has been studied by many authors [29-31] in the context of the magnetic field generation. For the amplification of the magnetic field itself the pseudoscalar vertex is not essential but it may lead to hypermagnetic flux lines are linked or twisted as originally discussed in [32]. The produced Chern-Simons condensates leads to a viable (but unconventional) mechanism for baryogenesis via hypermagnetic knots [32,33] which are characterized by an average magnetic gyrotropy [34]. These gyrotropic and helical fields play also a role in anomalous magnetohydrodynamics where the evolution of the magnetic fields at finite conductivity is analyzed in the presence of anomalous charges [35]. In the collisions of heavy ions this phenomenon is often dubbed chiral magnetic effect [36].

In this paper duality will be explicitly used to deduce the gauge power spectra not only during inflation but also during the subsequent decelerated expansion. We will show, in particular, that after the gauge coupling flattens out the late-time hypermagnetic power spectra outside the horizon in the radiation epoch are determined by the hyperelectric fields at the end of inflation whereas the opposite is true in the case of decreasing coupling. The obtained results suggest that a slightly blue hyperelectric spectrum during inflation may lead to a quasi-flat hypermagnetic spectrum prior to matter radiation equality and before the relevant wavelengths reenter the effective horizon. From the technical viewpoint these results will arise from the discussion of an appropriate transition matrix whose elements have well defined transformation properties under the duality symmetry and control the form of the late-time spectra. Using these results we shall investigate the magnetogenesis requirements as well as all other pertinent constraints; we shall conclude that large-scale magnetic fields can be generated during a quasi-de Sitter stage of expansion while the gauge coupling remains perturbative throughout all the stages of the dynamical evolution.

The layout of this paper is in short the following. In section 2 after introducing the necessary generalities, we shall discuss the duality symmetry both for the field equations and for the power spectra. At the end of the section we shall discuss two complementary (and dual) profiles for the evolution of the gauge couplings. In section 3 the gauge power spectra during inflation will be computed for typical wavelengths larger than the effective horizon and related via duality transformations. Using the overall continuity of the whole description the electric and the magnetic mode functions at the end of inflation will be explicitly related, in section 4 , to the gauge spectra in the radiation epoch via a transition matrix whose elements transform in a well defined way under duality. The gauge spectra will then be computed in several approximations schemes with particular attention to the relevant phenomenological regimes. In section 5 the magnetogenesis requirements will be examined in conjunction with the physical constraints. Section 6 contains our concluding remarks. To avoid lengthy digressions various technical results have been relegated to the appendices $\mathrm{A}-\mathrm{C}$.

\footnotetext{
${ }^{1}$ The Greek indices run over the four space-time dimensions while the Latin (lowercase) indices run over the three spatial dimensions. The signature of the four-dimensional metric will be mostly minus i.e. $(+,-,-,-)$. Finally the relation between the Riemann and the Ricci tensors will be chosen to be $R_{\mu \nu}=R^{\alpha}$
} 


\section{Equations of motion, duality and dynamical gauge couplings}

With the purpose of making the whole discussion self-contained, the essential notations will be introduced in the first part of this section while in the second part we shall examine the gauge power spectra in the light of the duality symmetry. In the last part of the section the physical aspects of the evolution of the gauge couplings will be specifically addressed.

\subsection{Notations, conventions and some general considerations}

The field content of the model may be different but it generally follows from a total action of the type:

$$
S_{\text {tot }}=S_{\text {grav }}+S_{\text {scalar }}+S_{\text {gauge }},
$$

where $S_{\text {gauge }}$ has been already introduced in equation (1.1) and, as already mentioned, $Y_{\mu \nu}$ and $\widetilde{Y}^{\mu \nu}$ denote, respectively, the gauge field strength and its dual:

$$
\widetilde{Y}^{\mu \nu}=\frac{1}{2} E^{\mu \nu \rho \sigma} Y_{\rho \sigma}, \quad E^{\mu \nu \rho \sigma}=\frac{\epsilon^{\mu \nu \rho \sigma}}{\sqrt{-G}} .
$$

In equation (2.2) $\epsilon^{\mu \nu \rho \sigma}$ denotes the totally antisymmetric symbol of Levi-Civita in four dimensions. In more explicit terms the gravitational and the scalar actions of equation (2.1) can be expressed as:

$$
\begin{aligned}
S_{\text {grav }} & =-\frac{1}{2 \ell_{P}^{2}} \int \mathrm{d}^{4} x \sqrt{-G} R, \\
S_{\text {scalar }} & =\int \mathrm{d}^{4} x \sqrt{-G}\left[\frac{1}{2} G^{\alpha \beta} \partial_{\alpha} \varphi \partial_{\beta} \varphi+\frac{1}{2} G^{\alpha \beta} \partial_{\alpha} \psi \partial_{\beta} \psi-W(\varphi, \psi)\right],
\end{aligned}
$$

where $\ell_{P}^{2}=1 / \bar{M}_{P}^{2}=8 \pi / M_{P}^{2}$ and $M_{P}=1.22 \times 10^{19} \mathrm{GeV}$ is the Planck mass. The notations of equation (2.3) are purposely schematic and $\varphi$ denotes the inflaton while $\psi$ is a spectator field. Various magnetogenesis models have been discussed in various contexts where $W(\varphi, \psi)$ has a well defined expression. For instance in the models of reference [23] $W$ is only function of $\varphi$ while an explicit magnetogenesis model based on spectator fields can be found in the last paper of reference [24]. With these specifications, the general equations derived from the actions (2.1)-(2.3) are:

$$
\begin{aligned}
& R_{\mu}^{\nu}=\ell_{P}^{2}\left[\partial_{\mu} \varphi \partial^{\nu} \varphi-W \delta_{\mu}^{\nu}+\mathcal{T}_{\mu}^{\nu}\right], \\
& G^{\alpha \beta} \nabla_{\alpha} \nabla_{\beta} \varphi+\frac{\partial W}{\partial \varphi}+\frac{1}{16 \pi} \frac{\partial \lambda}{\partial \varphi} Y_{\alpha \beta} Y^{\alpha \beta}=0, \\
& \nabla_{\alpha}\left(\lambda Y^{\alpha \beta}\right)=0, \quad \nabla_{\alpha} \widetilde{Y}^{\alpha \beta}=0 .
\end{aligned}
$$

In equation (2.4) $\mathcal{T}_{\mu}^{\nu}$ denotes the (traceless) energy-momentum tensor of the gauge fields whose explicit form is given by:

$$
\mathcal{T}_{\mu}^{\nu}=\frac{\lambda}{4 \pi}\left[-Y_{\mu \alpha} Y^{\nu \beta}+\frac{1}{4} \delta_{\mu}^{\nu} Y_{\alpha \beta} Y^{\alpha \beta}\right] .
$$

We shall be mostly concerned with conformally flat background geometries whose associated line element is:

$$
\mathrm{d} s^{2}=G_{\alpha \beta} \mathrm{d} x^{\alpha} \mathrm{d} x^{\alpha}=a^{2}(\tau)\left[\mathrm{d} \tau^{2}-\mathrm{d} \vec{x}^{2}\right],
$$


where $a(\tau)$ denotes the scale factor. In the geometry (2.8) the various components of $\mathcal{T}_{\mu}{ }^{\nu}$ defined in equation (2.7) are:

$$
\begin{aligned}
& \mathcal{T}_{0}{ }^{0}=\rho_{B}+\rho_{E}, \quad \mathcal{T}_{0}{ }^{i}=\frac{1}{4 \pi a^{4}}(\vec{E} \times \vec{B})^{i}, \\
& \mathcal{T}_{i}{ }^{j}=-\left(p_{E}+p_{B}\right) \delta_{i}^{j}+\Pi_{E i}^{j}+\Pi_{B i}^{j} .
\end{aligned}
$$

In equation (2.9) we introduced the energy density, the pressure and the anisotropic stresses of the hypermagnetic and hyperelectric fields:

$$
\begin{array}{rlrl}
\rho_{B} & =\frac{B^{2}}{8 \pi a^{4}}, \quad \rho_{E}=\frac{E^{2}}{8 \pi a^{4}}, & p_{B}=\frac{\rho_{B}}{3}, & p_{E}=\frac{\rho_{E}}{3}, \\
\Pi_{E i}^{j}=\frac{1}{4 \pi a^{4}}\left(E_{i} E^{j}-\frac{E^{2}}{3} \delta_{i}^{j}\right), & \Pi_{B i}^{j}=\frac{1}{4 \pi a^{4}}\left(B_{i} B^{j}-\frac{B^{2}}{3} \delta_{i}^{j}\right),
\end{array}
$$

where $E^{2}=\vec{E} \cdot \vec{E}$ and $B^{2}=\vec{B} \cdot \vec{B}$. Equations (2.9)-(2.11) are expressed in terms of $\vec{E}$ and $\vec{B}$, i.e. the comoving hyperelectric and hypermagnetic fields. These rescaled quantities are actually the normal modes of the system and are related to the physical fields as:

$$
\vec{E}=a^{2} \sqrt{\lambda} \vec{E}^{\text {(phys) }}, \quad \vec{B}=a^{2} \sqrt{\lambda} \vec{B}^{\text {(phys) }} .
$$

The components of the field strengths can be directly expressed in terms of the components of the physical fields; so for instance, in terms of $\epsilon^{i j k}$ (i.e. the Levi-Civita symbol in three dimensions) we have

$$
Y_{i 0}=-a^{2} E_{i}^{\text {(phys) }}, \quad Y^{i j}=-\epsilon^{i j k} B_{k}^{\text {(phys) }} / a^{2},
$$

and similarly for the dual strength. The physical fields are essential for the discussion of the actual magnetogenesis constraints but the evolution equations of (2.6) are simpler in terms of the comoving fields:

$$
\begin{aligned}
& \vec{\nabla} \cdot(\sqrt{\lambda} \vec{E})=0, \quad \vec{\nabla} \cdot\left(\frac{\vec{B}}{\sqrt{\lambda}}\right)=0, \\
& \partial_{\tau}(\sqrt{\lambda} \vec{E})=\vec{\nabla} \times(\sqrt{\lambda} \vec{B}), \quad \partial_{\tau}\left(\frac{\vec{B}}{\sqrt{\lambda}}\right)=-\vec{\nabla} \times\left(\frac{\vec{E}}{\sqrt{\lambda}}\right) .
\end{aligned}
$$

Equations (2.14) and (2.15) have been written in the general case where $\lambda$ can be inhomogeneous even if, as we shall see in the last part of this section, the gauge coupling will always be considered to be time-dependent but homogeneous. Furthermore equations (2.14) and (2.15) under the following duality transformation:

$$
\sqrt{\lambda} \rightarrow \frac{1}{\sqrt{\lambda}}, \quad \vec{B} \rightarrow \vec{E}, \quad \vec{E} \rightarrow-\vec{B} .
$$

All the mode functions and power spectra obtained by the simultaneous evolution of the geometry and of the gauge coupling must be consistent with equation (2.16) both during the inflationary phase and in the subsequent decelerated stages of expansion before the given scale reenters the effective horizon. The gauge coupling $e(\lambda)$ is related to the inverse of $\sqrt{\lambda}$ :

$$
S_{\text {gauge }}=-\frac{1}{4} \int \mathrm{d}^{4} x \frac{\sqrt{-G}}{e^{2}} Y_{\mu \nu} Y^{\mu \nu}, \quad e^{2}=\frac{4 \pi}{\lambda} .
$$


Thus the gauge coupling increases when $\lambda$ decreases and vice versa.

\subsection{Quantization and canonical form of the power spectra}

Since we are going to discuss the parametric amplification of the quantum fluctuations of the gauge fields the classical evolution summarized by equations (2.14) and (2.15) must be complemented by the corresponding quantum treatment. From the semi-classical viewpoint this process can be viewed as the conversion of traveling waves into standing waves; the same phenomenon occurs for the scalar and tensor modes of the geometry [22] and it leads to the so-called Sakharov oscillations [37] (see also [38-40]). In the present context the duality symmetry explicitly relates the Sakharov oscillations of the hypermagnetic [i.e. $f_{k, \alpha}(\tau)$ ] and hyperelectric mode functions [i.e. $\left.g_{k, \alpha}(\tau)\right]$ entering the expressions of the corresponding field operators:

$$
\begin{aligned}
& \hat{B}_{i}(\tau, \vec{x})=-\frac{i \epsilon_{m n i}}{(2 \pi)^{3 / 2}} \sum_{\alpha=\oplus, \otimes} \int \mathrm{d}^{3} k k_{m} e_{n}^{(\alpha)}(\hat{k})\left[f_{k, \alpha}(\tau) \hat{a}_{\vec{k}, \alpha} \mathrm{e}^{-\mathrm{i} \vec{k} \cdot \vec{x}}-f_{k, \alpha}^{*}(\tau) \hat{a}_{\vec{k}, \alpha}^{\dagger} \mathrm{e}^{\mathrm{i} \vec{k} \cdot \vec{x}}\right], \\
& \hat{E}_{i}(\tau, \vec{x})=-\frac{1}{(2 \pi)^{3 / 2}} \sum_{\alpha=\oplus, \otimes} \int \mathrm{d}^{3} k e_{i}^{(\alpha)}(\hat{k})\left[g_{k \alpha}(\tau) \hat{a}_{\vec{k}, \alpha} \mathrm{e}^{-\mathrm{i} \vec{k} \cdot \vec{x}}+g_{k, \alpha}^{*}(\tau) \hat{a}_{\vec{k}, \alpha}^{\dagger} \mathrm{e}^{\mathrm{i} \vec{k} \cdot \vec{x}}\right] .
\end{aligned}
$$

In equations (2.18) and (2.19) the two vector polarizations are directed along the orthogonal unit vectors $\hat{e}_{\oplus}$ and $\hat{e}_{\otimes}$ that are also orthogonal to $\hat{k}$ (i.e. $\hat{k} \cdot \hat{e}_{\alpha}=0$ ). In equation (2.19) $\hat{a}_{\vec{k}, \alpha}$ and $\hat{a}_{\vec{k}, \alpha}^{\dagger}$ are the creation and annihilation operators obeying, within the present notations, $\left[\hat{a}_{\vec{q}, \alpha}, \hat{a}_{\vec{p}, \beta}^{\dagger}\right]=\delta^{(3)}(\vec{q}-\vec{p}) \delta_{\alpha \beta}$. In equations (2.18) and (2.19) the sum is performed over the physical polarizations $e_{i}^{(\alpha)}(\hat{k})$ while the mode functions $f_{k, \alpha}$ and $g_{k, \alpha}$ obey, in the absence of conductivity, the following pair of equations:

$$
f_{k, \alpha}^{\prime}=g_{k, \alpha}+\mathcal{F} f_{k, \alpha}, \quad g_{k, \alpha}^{\prime}=-k^{2} f_{k, \alpha}-\mathcal{F} g_{k, \alpha}, \quad \mathcal{F}=\frac{\sqrt{\lambda}^{\prime}}{\sqrt{\lambda}}
$$

where the prime denotes a derivation with respect to the conformal time coordinate $\tau$. The mode functions in equation (2.20) must also be correctly normalized so that the Wronskian of any solution must satisfy for each polarization:

$$
f_{k, \alpha}(\tau) g_{k, \alpha}^{*}(\tau)-f_{k, \alpha}^{*}(\tau) g_{k, \alpha}(\tau)=i .
$$

The field operators of equations (2.18) and (2.19) can be represented in Fourier space as ${ }^{2}$

$$
\begin{aligned}
& \hat{B}_{j}(\vec{p}, \tau)=-i p_{m} \epsilon_{m n j} \sum_{\beta}\left[e_{n}^{\beta}(\hat{p}) \hat{a}_{\vec{p}, \beta} f_{q, \beta}(\tau)+e_{n}^{\alpha}(-\hat{p}) \hat{a}_{-\vec{p}, \beta}^{\dagger} f_{p, \beta}^{*}(\tau)\right], \\
& \hat{E}_{i}(\vec{q}, \tau)=\sum_{\alpha}\left[e_{i}^{\alpha}(\hat{q}) \hat{a}_{\vec{q}, \alpha} g_{q, \alpha}(\tau)+e_{i}^{\alpha}(-\hat{q}) \hat{a}_{-\vec{q}, \alpha}^{\dagger} g_{q, \alpha}^{*}(\tau)\right] .
\end{aligned}
$$

Therefore the corresponding two-point functions in Fourier space become:

$$
\left\langle\hat{B}_{i}(\vec{k}, \tau) \hat{B}_{j}(\vec{p}, \tau)\right\rangle=\frac{2 \pi^{2}}{k^{3}} P_{B}(k, \tau) p_{i j}(\hat{k}) \delta^{(3)}(\vec{k}+\vec{p}),
$$
${ }^{2}$ Equation (2.22) follows from equations (2.18) and (2.19) by recalling that $\hat{B}_{j}(\vec{p}, \tau)=\int \mathrm{d}^{3} x \hat{B}_{j}(\vec{x}, \tau) \mathrm{e}^{\mathrm{i} \vec{p} \cdot \vec{x}} /(2 \pi)^{3 / 2}$ and
that $\hat{E}_{i}(\vec{q}, \tau)=\int \mathrm{d}^{3} x \hat{E}_{i}(\vec{x}, \tau) \mathrm{e}^{\mathrm{i} q \vec{x} \cdot \vec{x}} /(2 \pi)^{3 / 2}$. 


$$
\left\langle\hat{E}_{i}(\vec{k}, \tau) \hat{E}_{j}(\vec{p}, \tau)\right\rangle=\frac{2 \pi^{2}}{k^{3}} P_{E}(k, \tau) p_{i j}(\hat{k}) \delta^{(3)}(\vec{k}+\vec{p}),
$$

where $p(\hat{k})=\left(\delta_{i j}-\hat{k}_{i} \hat{k}_{j}\right) ; P_{B}(k, \tau)$ and $P_{E}(k, \tau)$ are the comoving magnetic and electric power spectra, respectively ${ }^{3}$ :

$$
\begin{aligned}
& P_{B}(k, \tau)=\frac{k^{5}}{4 \pi^{2}} \sum_{\alpha=\oplus, \otimes}\left|f_{k, \alpha}(\tau)\right|^{2} \equiv \frac{k^{5}}{2 \pi^{2}}\left|f_{k}(\tau)\right|^{2}, \\
& P_{E}(k, \tau)=\frac{k^{3}}{4 \pi^{2}} \sum_{\alpha=\oplus, \otimes}\left|g_{k, \alpha}(\tau)\right|^{2} \equiv \frac{k^{3}}{2 \pi^{2}}\left|g_{k}(\tau)\right|^{2} .
\end{aligned}
$$

For the present purposes it will be important to distinguish the comoving from the physical power spectra: while the comoving power spectra are obtained from the comoving field operators $\hat{E}_{i}$ and $\hat{B}_{i}$, the physical power spectra follow from the corresponding physical fields $\hat{E}_{i}^{\text {(phys) }}$ and $\hat{B}_{i}^{\text {(phys) }}$ defined in equation (2.12); the relation between the physical and the comoving power spectra is therefore given by:

$$
P_{B}^{\text {(phys) }}(k, \tau)=\frac{P_{B}(k, \tau)}{\lambda(\tau) a^{4}(\tau)}, \quad P_{E}^{\text {(phys) }}(k, \tau)=\frac{P_{E}(k, \tau)}{\lambda(\tau) a^{4}(\tau)} .
$$

The phenomenological requirements (e.g. the magnetogenesis constraints to be discussed in section 5) must be typically expressed in terms of the physical spectra. Occasionally this distinction has not been clearly spelled out and the consequences have been confusing, as we shall remark later on. Let us conclude this discussion by recalling that the two coupled firstorder equations given in (2.20) can be transformed in two (decoupled) second-order differential equations:

$$
f_{k, \alpha}^{\prime \prime}+\left[k^{2}-\frac{\sqrt{\lambda}^{\prime \prime}}{\sqrt{\lambda}}\right] f_{k, \alpha}=0, \quad g_{k, \alpha}^{\prime \prime}+\left[k^{2}-\sqrt{\lambda}\left(\frac{1}{\sqrt{\lambda}}\right)^{\prime \prime}\right] g_{k, \alpha}=0,
$$

which have the same content of (2.20) provided the initial conditions are correctly imposed by taking into account that $g_{k, \alpha}$ is ultimately determined from $f_{k, \alpha}$ and its derivative as $g_{k, \alpha}=f_{k \alpha}^{\prime}-\mathcal{F} f_{k \alpha}$. Equations (2.20)-(2.28) are invariant under the following duality transformations ${ }^{4}$ :

$$
\sqrt{\lambda} \rightarrow 1 / \sqrt{\lambda}, \quad f_{k, \alpha} \rightarrow g_{k, \alpha} / k, \quad g_{k, \alpha} \rightarrow-k f_{k, \alpha} .
$$

\subsection{Spectral energy density and backreaction constraint}

Since the amplification of the gauge fields takes place in a homogeneous and isotropic background geometry (see equation (2.8)) the corresponding energy density must not exceed the critical energy density $\rho_{\text {crit }}=3 \bar{M}_{P}^{2} H^{2}$ where $H$ is the Hubble rate. The averaged energy density of the gauge fields follows from equations (2.23) and (2.24) and from the definitions of $\rho_{B}$ and

\footnotetext{
${ }^{3}$ If the mode functions for the two polarizations coincide the sums appearing in equations (2.25) and (2.26) can be performed trivially since $f_{k \oplus}=f_{k \otimes}=f_{k}$ and similarly for the hyperelectric mode function.

${ }^{4}$ In connection with equations (2.20) and (2.29) we recall that $\mathcal{F}=\sqrt{\lambda} / \sqrt{\lambda}$; this means that for $\sqrt{\lambda} \rightarrow 1 / \sqrt{\lambda}$, $\mathcal{F} \rightarrow-\mathcal{F}$.
} 


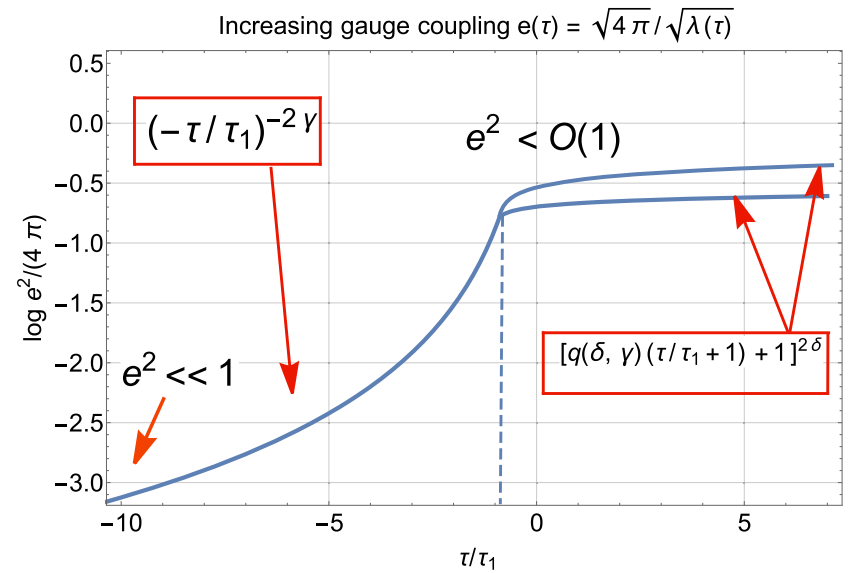

Figure 1. The logic and the main notations employed for the dynamical description of an increasing gauge coupling. The two different curves for $\tau \geqslant-\tau_{1}$ correspond to different values of $\delta \ll 1$. Note that $q(\delta, \gamma)=\delta / \gamma$ (see also equation (A.3)).

$\rho_{E}$ appearing in equations (2.9)-(2.11). Thanks to equations (2.25) and (2.26) the final result is:

$$
\left\langle\hat{\rho}_{Y}\right\rangle=\left\langle\hat{\rho}_{B}\right\rangle+\left\langle\hat{\rho}_{E}\right\rangle=\frac{1}{4 \pi a^{4}(\tau)} \int \frac{\mathrm{d} k}{k}\left[P_{E}(k, \tau)+P_{B}(k, \tau)\right] .
$$

In terms of the physical power spectra of equation (2.27) the result (2.30) becomes:

$$
\left\langle\hat{\rho}_{Y}\right\rangle=\frac{\lambda(\tau)}{4 \pi} \int \frac{\mathrm{d} k}{k}\left[P_{E}^{\text {(phys) }}(k, \tau)+P_{B}^{\text {(phys) }}(k, \tau)\right] .
$$

To compare energy density of the parametrically amplified gauge fields with the energy density of the background geometry we introduce the spectral energy density in critical units:

$$
\Omega_{Y}=\frac{1}{\rho_{\text {crit }}} \frac{\mathrm{d}\langle\hat{\rho}\rangle}{\mathrm{d} \ln k}=\frac{2}{3 H^{2} M_{P}^{2} a^{4}}\left[P_{E}(k, \tau)+P_{B}(k, \tau)\right] .
$$

To guarantee the absence of dangerous backreaction effects $\Omega_{Y}(k, \tau)$ must always be much smaller 1 throughout all the stages of the evolution and for all relevant scales; this requirement must be separately verified both during and after inflation.

\subsection{Gauge couplings and their continuity}

2.4.1. Increasing gauge coupling. In figure 1 the profile describing the evolution of the gauge coupling is illustrated together with the main notations employed throughout the discussion. During the inflationary phase (i.e. for $\tau \leqslant-\tau_{1}$ in figure 1) $\gamma$ describes the rate of increase of the gauge coupling in the conformal time parametrization while $\delta$ controls the evolution during the post-inflationary stage of expansion when the gauge coupling flattens out:

$$
\begin{aligned}
& \sqrt{\lambda}=\sqrt{\lambda_{1}}\left(-\frac{\tau}{\tau_{1}}\right)^{\gamma}, \quad \tau \leqslant-\tau_{1}, \\
& \sqrt{\lambda}=\sqrt{\lambda_{1}}\left[\frac{\gamma}{\delta}\left(\frac{\tau}{\tau_{1}}+1\right)+1\right]^{-\delta}, \quad \tau \geqslant-\tau_{1} .
\end{aligned}
$$




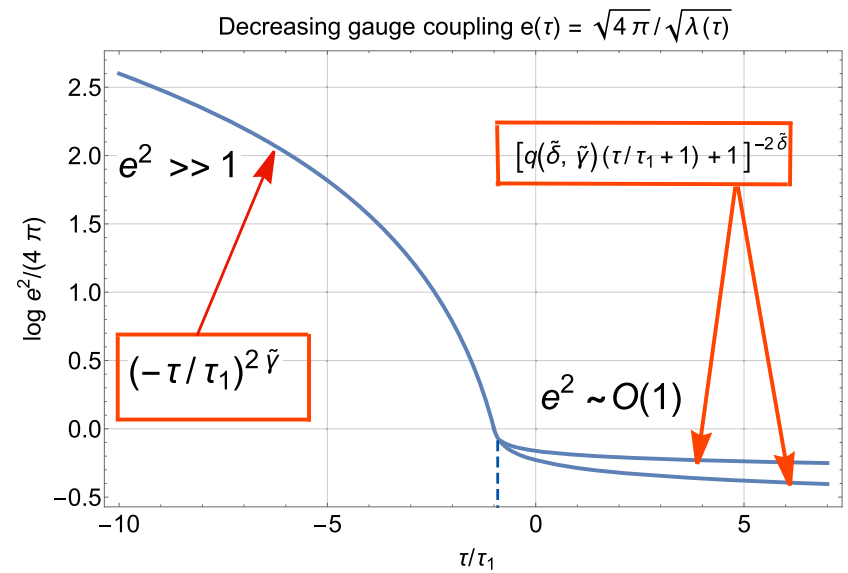

Figure 2. We illustrate the main notations employed when discussing the case of the decreasing gauge coupling. As in the case of figure 1 the two different curves for $\tau \geqslant-\tau_{1}$ correspond to different values of $\widetilde{\delta} \ll 1$. Note that $q(\widetilde{\delta}, \widetilde{\gamma})=\widetilde{\delta} / \widetilde{\gamma}$ (see also equation (B.4)).

The explicit form of equations (2.33) and (2.34) is dictated by the continuity of $\sqrt{\lambda}$ and of $\sqrt{\lambda}^{\prime}$; furthermore the physical range of $\gamma$ and $\delta$ is given by:

$$
\gamma>0, \quad \text { and } \quad 0 \leqslant \delta \ll \gamma
$$

For short the limit $\delta \rightarrow 0$ shall be referred to as the sudden approximation while the smooth approximation corresponds to the case of equation (2.35) where $0 \leqslant \delta \ll \gamma$. As we shall see it will always be possible to derive the results of the sudden approximation by taking the limit $\delta \rightarrow 0$ from the formulas valid in the case of the smooth approximation. The continuity of the magnetic and electric mode functions introduced in equations (2.18) and (2.19) and obeying equations (2.20) and (2.21) rely however on the continuity of $\sqrt{\lambda}$ and its derivative. If we would simply assume that $\sqrt{\lambda}$ is constant the conformal time derivative will have a jump discontinuity and equation (2.28) will develop a singularity either in $\sqrt{\lambda}^{\prime \prime} / \sqrt{\lambda}$ or in $(1 / \sqrt{\lambda})^{\prime \prime} \sqrt{\lambda}$. Since the present approach guarantees the continuity of gauge mode functions the related power spectra will also be continuous. Note that the numerical value of $\lambda_{1}$ may well coincide with 1 but it could also be slightly larger than 1 since this range is compatible with a gauge coupling that is perturbative for $\tau=-\tau_{1}$, as illustrated in figure 1 .

In the geometry of equation (2.8) the background equations derived from equations (2.4) and (2.5) are:

$$
3 \bar{M}_{P}^{2} \mathcal{H}^{2}=\frac{1}{2} \varphi^{\prime 2}+a^{2} W(\varphi), \quad 2 \bar{M}_{P}^{2}\left(\mathcal{H}^{2}-\mathcal{H}^{\prime}\right)=\varphi^{\prime 2}, \varphi^{\prime \prime}+2 \mathcal{H} \varphi^{\prime}+\frac{\partial W}{\partial \varphi} a^{2}=0,
$$

where, as already mentioned, the prime denotes a derivation with respect to the conformal time coordinate $\tau$; furthermore $\mathcal{H}=(\ln a)^{\prime}=a H$ where $H=\dot{a} / a$ is the conventional Hubble rate and the overdot denotes a derivation with respect to the cosmic time coordinate $t$. The slow roll approximation specifies the evolution during the inflationary phase where the parameters $\epsilon, \eta$ and $\bar{\eta}$ are all much smaller than 1 and eventually get to 1 when inflation ends. The definitions 
of the slow roll parameters within the notations of this paper are as follows:

$$
\epsilon=-\frac{\dot{H}}{H^{2}}=\frac{\bar{M}_{P}^{2}}{2}\left(\frac{W_{, \varphi}}{W}\right)^{2}, \quad \eta=\frac{\ddot{\varphi}}{H \dot{\varphi}}, \quad \bar{\eta}=\bar{M}_{P}^{2}\left(\frac{W_{, \varphi \varphi}}{W}\right),
$$

note that $W_{, \varphi}$ and $W_{, \varphi \varphi}$ are shorthand notations for the first and second derivatives of the potential $W(\varphi)$ with respect to $\varphi$. The slow roll parameters $\eta, \bar{\eta}$ and $\epsilon$ are not independent and their mutual relation, i.e. $\eta=\epsilon-\bar{\eta}$, follows from the slow roll version of equation (2.36) written in the cosmic time coordinate $t$ :

$$
3 H \dot{\varphi}+\frac{\partial W}{\partial \varphi}=0, \quad 3 \bar{M}_{P}^{2} H^{2}=W, \quad 2 \bar{M}_{P}^{2} \dot{H}=-\dot{\varphi}^{2} .
$$

We shall be assuming that the inflationary stage of expansion takes place for $\tau \leqslant-\tau_{1}$ :

$$
\mathcal{H}=a H=-\frac{1}{(1-\epsilon) \tau}, \quad \epsilon=-\frac{\dot{H}}{H^{2}} \ll 1,
$$

whereas after inflation (i.e. for $\tau \geqslant-\tau_{1}$ ) the background will be decelerated and dominated by radiation. The continuity of the scale factor demands:

$$
\begin{aligned}
& a_{\mathrm{inf}}(\tau)=\left(-\frac{\tau}{\tau_{1}}\right)^{-\beta}, \quad \tau \leqslant-\tau_{1}, \\
& a_{\mathrm{rad}}(\tau)=\frac{\tau+(\beta+1) \tau_{1}}{\tau_{1}}, \quad \tau \geqslant-\tau_{1} .
\end{aligned}
$$

with $\beta \simeq 1 /(1-\epsilon)$. Both equations (2.39) and (2.40) assume that $\epsilon$ changes very slowly during inflation. The continuity of the scale factor and of its conformal time derivative also imply the continuity of the extrinsic curvature whose background value is given by $\bar{K}_{i j}=-a \mathcal{H} \delta_{i j}$.

For a correct treatment of the problem the continuity of the extrinsic curvature is essential. However various other effects could be always added with the purpose of providing a more accurate description of the transition regime. For instance it could be observed that the slowroll approximation breaks down at the end of inflation so that $\epsilon$ is not truly constant across the transition. Another possibility is represented by the dynamics of reheating. These situations will affect the maximal frequency of the spectrum which is therefore rather difficult to predict with great accuracy. This situation is actually analog to what happens in the case of the spectrum of relic gravitons [45]. In analogy with the graviton spectrum we shall therefore parametrize the maximal wavenumber as $k_{\max }=\zeta a_{1} H_{1}$ where $\zeta$ is a numerical factor which may even be, in some cases, dependent upon the wavenumber. The impact of the breakdown of the slowroll approximation on the maximal frequency of the spectrum has been discussed in [46]. In the present case these considerations are anyway immaterial since the typical wavenumbers relevant for magnetogenesis are typically much smaller i.e. $\mathcal{O}\left(\mathrm{Mpc}^{-1}\right)$ as we shall see.

In a complementary perspective it should also be mentioned that the maximal frequency of the spectrum could be affected by the details of the reheating dynamics. As already suggested in the previous paragraph the continuity of the extrinsic curvature is an excellent starting point also in this case. More detailed models of reheating could be certainly useful and might affect the magnetogenesis scenarios (as suggested in [47]). In this paper we adopted the approximation of the sudden reheating which is also the one often employed in order to set limits on the tensor to scalar ratio and on the total number of inflationary $e$-folds. We shall however relax this approximation in phenomenological discussion of section 5 . 
2.4.2. Decreasing gauge coupling. For the purposes of the present analysis it is useful to complement the timeline of figure 1 with figure 2 describing the dual situation where the gauge coupling is initially much larger than 1 . In the situation illustrated by figure 2 the continuity of $\sqrt{\lambda}$ and $\sqrt{\lambda}^{\prime}$ imply the following parametrization

$$
\begin{aligned}
& \sqrt{\lambda}=\sqrt{\lambda_{1}}\left(-\frac{\tau}{\tau_{1}}\right)^{-\widetilde{\gamma}}, \quad \tau<-\tau_{1}, \\
& \sqrt{\lambda}=\sqrt{\lambda_{1}}\left[\frac{\widetilde{\gamma}}{\widetilde{\delta}}\left(\frac{\tau}{\tau_{1}}+1\right)+1\right]^{\tilde{\delta}}, \quad \tau \geqslant-\tau_{1},
\end{aligned}
$$

the physical region of the parameters is given by:

$$
\widetilde{\gamma}>0, \quad \widetilde{\delta} \geqslant 0, \quad \text { and } \quad 0<\widetilde{\delta} \ll \widetilde{\gamma}
$$

The limit $\widetilde{\delta} \rightarrow 0$ denotes again the sudden approximation while the smooth approximation holds for $\widetilde{\delta} \ll 1$ or, which is the same, $\widetilde{\delta} \ll \widetilde{\gamma}$ since we shall assume throughout that $\widetilde{\gamma}$ is of order 1. As in the case of increasing gauge coupling (see equations (2.33) and (2.34)), equations (2.41) and (2.42) will be complemented by the smooth evolution of the geometry illustrated in equation (2.40). The dual profiles of figures 1 and 2 are not physically equivalent. If we consider a certain reference time $\tau=-\tau_{i}$ close to the onset of the inflationary phase, we will have, according to equation (2.41) that

$$
\sqrt{\lambda_{i}}=\sqrt{\lambda_{1}}\left(\frac{a_{i}}{a_{f}}\right)^{\tilde{\gamma}} \ll 1 \Rightarrow e\left(\tau_{i}\right)=\frac{\sqrt{4 \pi}}{\sqrt{\lambda_{i}}} \gg 1,
$$

where, by definition, $\lambda_{i}=\lambda\left(-\tau_{i}\right)$; in equation (2.44) we traded the conformal time for the scale factors by using equation (2.40) in the limit $\epsilon \ll 1$. Note in fact that $\left(a_{i} / a_{f}\right)^{\widetilde{\gamma}}=e^{-N \widetilde{\gamma}} \ll 1$ where $N$ is the total number of inflationary $e$-folds. Since $N=\mathcal{O}(60)$ (or larger) we have that $\sqrt{\lambda_{i}}$ will be $\mathcal{O}\left(10^{-60}\right)$ (or smaller). Equation (2.44) implies that the evolution of the gauge coupling starts from a non-perturbative regime unless $\sqrt{\lambda_{1}}$ is extremely large: only in this way we would have $\sqrt{\lambda_{i}}=\mathcal{O}(1)$. Whenever $\sqrt{\lambda_{1}} \gg 1$ the gauge coupling will be extremely minute at the end of inflation and this is at odds with the fact that during the decelerated stage of expansion we would like to have $e^{2}=\mathcal{O}\left(10^{-2}\right)$ but not much smaller. Furthermore, as we shall see in section 5 , the physical power spectra are suppressed as $\lambda_{1}^{-1}$ and this will make their contribution marginal for the phenomenological implications. A possibility suggested in reference [44] has been that the $\sqrt{\lambda}$ increases during inflation, decreases sharply during reheating, and then flattens out again. This suggestion is often assumed by various authors but rarely justified.

\section{Inflationary gauge spectra and their constraints}

The qualitative description of large-scale cosmological perturbations suggests that a given wavelength exits the Hubble radius at some typical conformal time during an inflationary stage of expansion and approximately reenters at $\tau_{k} \sim 1 / k$, when the Universe still expands but in a decelerated manner. By a mode being beyond the horizon we only mean that the physical wavenumber is much less than the expansion rate: this does not necessarily have anything to do with causality $[22,41]$. Similarly the physical wavenumbers of the hyperelectric and hypermagnetic fields can be much smaller than the rate of variation of the gauge coupling which now plays the role of the effective horizon. During inflation the relevant regime will be the 
one where the wavelengths of the gauge fluctuations are inside the effective horizon which means that $k / \mathcal{F} \simeq k \tau \ll 1$. As long as $k \tau<1$ duality will be a valid symmetry but as soon as $k \tau_{k} \sim 1$ the electric spectra will be suppressed by conductivity and the gauge spectra will not be related by duality (see in this respect section 5). Not all the wavelengths that are larger than the effective horizon will reenter at the end of inflation (i.e. for $\tau=-\tau_{1}$ ) but throughout the whole radiation phase. Thus the gauge spectra for wavelengths larger than the effective horizon during inflation do not necessarily coincide with the gauge spectra when the given mode reenters (see, in this respect, section 4).

The logic of this section will be to compute the gauge spectra during inflation for the profiles schematically introduced in figures 1 and 2 . We shall then demonstrate that the gauge power spectra in the two cases are explicitly related by duality. This will mean, for instance, that during inflation the generation of potentially scale-invariant hyperelectric spectrum is only compatible with a phase where the gauge coupling decreases while a flat hypermagnetic spectrum may only arise when the gauge coupling decreases.

\subsection{Increasing gauge coupling}

When $\sqrt{\lambda}$ is given by (2.33) the solution of equation (2.20) compatible with the Wronskian normalization dictated by equation (2.21) is given by ${ }^{5}$ :

$$
\begin{aligned}
& f_{k}(\tau)=\frac{N_{\mu}}{\sqrt{2 k}} \sqrt{-k \tau} H_{\mu}^{(1)}(-k \tau), \quad \mu=\left|\gamma-\frac{1}{2}\right|, \\
& g_{k}(\tau)=N_{\mu} \sqrt{\frac{k}{2}} \sqrt{-k \tau} H_{\mu+1}^{(1)}(-k \tau), \quad \gamma>\frac{1}{2}, \\
& g_{k}(\tau)=-N_{\mu} \sqrt{\frac{k}{2}} \sqrt{-k \tau} H_{\mu-1}^{(1)}(-k \tau), \quad 0<\gamma<\frac{1}{2} .
\end{aligned}
$$

In general $H_{\nu}^{(1)}(z)$ will denote the Hankel functions first kind $[42,43]$ with argument $z$ and index $\nu ; N_{\nu}$ is a complex number whose phase is required for a correct asymptotic normalization of the mode functions:

$$
H_{\nu}^{(1)}(z)=J_{\nu}(z)+\mathrm{i} Y_{\nu}(z), \quad N_{\nu}=\sqrt{\frac{\pi}{2}} \mathrm{e}^{\mathrm{i} \pi(2 \nu+1) / 4} .
$$

Throughout the whole discussion we shall assume, as in the standard theory of Hankel functions $[42,43]$ that $\nu$ is real and positive semi-definite:

$$
\operatorname{Re} \nu \geqslant 0, \quad \operatorname{Im} \nu=0, \quad H_{-\nu}^{(1)}(z)=\mathrm{e}^{\mathrm{i} \pi \nu} H_{\nu}^{(1)}(z), \quad H_{-\nu}^{(2)}(z)=\mathrm{e}^{-\mathrm{i} \pi \nu} H_{\nu}^{(2)}(z) .
$$

Inserting the correctly normalized mode functions of equations (3.1)-(3.3) into equations (2.25) and (2.26) the gauge spectra for $\tau \leqslant-\tau_{1}$ turn out to be ${ }^{6}$ :

$$
P_{B}(k, \tau)=\frac{a^{4} H^{4}}{8 \pi}(-k \tau)^{5}\left|H_{\mu}^{(1)}(-k \tau)\right|^{2} \rightarrow a^{4} H^{4} D(\mu)|k \tau|^{5-2 \mu},
$$

${ }^{5}$ For $\gamma \rightarrow 1 / 2, \mu \rightarrow 0$ and, in this limit, equations (3.2) and (3.3) coincide exactly since $H_{-1}^{(1)}(z)=\mathrm{e}^{\mathrm{i} \pi} H_{1}^{(1)}(z)$. See also equation (3.5).

${ }^{6} \mathrm{~A}$ term $|1-\epsilon|^{4}$ has been omitted in the prefactors since it coincides with 1 for $\epsilon \ll 1$. 


$$
\begin{aligned}
P_{E}(k, \tau)= & \frac{a^{4} H^{4}}{8 \pi}(-k \tau)^{5}\left|H_{\mu+1}^{(1)}(-k \tau)\right|^{2} \\
& \rightarrow a^{4} H^{4} D(\mu+1)|k \tau|^{5-2(\mu+1)}, \quad \gamma>1 / 2, \\
P_{E}(k, \tau)= & \frac{a^{4} H^{4}}{8 \pi}(-k \tau)^{5}\left|H_{\mu-1}^{(1)}(-k \tau)\right|^{2} \\
& \rightarrow a^{4} H^{4} D(|\mu-1|)|k \tau|^{5-2|\mu-1|}, \quad 0<\gamma<1 / 2,
\end{aligned}
$$

where we introduced the function:

$$
D(x)=2^{2 x-3} \frac{\Gamma^{2}(x)}{\pi^{3}}
$$

that will be used throughout the whole paper exactly with the same meaning; the function $D(x)$ arises when the gauge spectra are evaluated outside the effective horizon (i.e. for $|k \tau|<1$ ) and the corresponding Hankel functions are estimated using their limit for small arguments $[42,43]$. Note, in this respect, that $(-k \tau)$ can also be expressed as ${ }^{7}$

$$
(-k \tau)=\frac{k}{(1-\epsilon) a H} \simeq \frac{k}{a H}=\frac{k}{a_{1} H} e^{-N}, \quad \epsilon \ll 1, N=\ln \left(\frac{a}{a_{1}}\right) .
$$

Since, by definition, $\mu=|\gamma-1 / 2|$ in terms of $\gamma$ the comoving power spectra of equations (3.6)-(3.8) become:

$$
\begin{aligned}
& P_{B}(k, \tau)=a^{4} H^{4} D(|\gamma-1 / 2|)|k \tau|^{5-|2 \gamma-1|}, \\
& P_{E}(k, \tau)=a^{4} H^{4} D(\gamma+1 / 2)|k \tau|^{4-2 \gamma} .
\end{aligned}
$$

The derivation of equation (3.11) from (3.6) is immediate from the definition of $\mu$ in terms of $\gamma$ (i.e. $\mu=|\gamma-1 / 2|$ ). On the contrary equation (3.12) is the common expression of equations (3.7) and (3.8). In fact, in equation (3.7) $\mu=\gamma-1 / 2$ (and therefore $\mu+1=$ $\gamma+1 / 2$ ), conversely in (3.8) $\mu=1 / 2-\gamma$ and $|\mu-1|$ always equals $\gamma+1 / 2$. After direct insertion of equations (3.11) and (3.12) into equation (2.32) the spectral energy density for $\tau \leqslant-\tau_{1}$ is

$$
\Omega_{Y}(k, \tau)=\frac{2}{3}\left(\frac{H}{M_{P}}\right)^{2}\left[D_{B}(|\gamma-1 / 2|)|k \tau|^{5-|2 \gamma-1|}+D_{E}(\gamma+1 / 2)|k \tau|^{4-2 \gamma}\right] .
$$

The spectral energy density of equation (3.13) must be always subcritical (i.e. $\Omega_{Y}(k, \tau) \ll 1$ ) for $\tau \leqslant-\tau_{1}$ and $|k \tau| \leqslant 1$; this requirement is not always satisfied even if, during the inflationary phase, $H \ll M_{P}$. The first term inside the square bracket at the right-hand side of equation (3.13) denotes the magnetic contribution while the second term is the electric result ${ }^{8}$. By looking together at equations (3.11)-(3.13) the following conclusions naturally emerge:

${ }^{7} N$ denotes the total number of inflationary $e$-folds and has nothing to do with the normalization $N_{\mu}$ of the mode functions.

${ }^{8}$ To stress this we just added a subscript to the function $D(x)$ (by writing $D_{B}(x)$ and $D_{E}(x)$ ) even if the definition of $D(x)$ (see after equation (3.8)) is the same in both cases. 
- If $\gamma=2$ the hyperelectric spectrum is exactly scale-invariant while the magnetic spectrum is steeply increasing [i.e. $P_{B}(k, \tau) \propto|k \tau|^{2}$ for $k \tau \ll 1$ ] so that the condition $\Omega_{Y}(k, \tau) \ll 1$ is safely satisfied;

- If $1 / 2<\gamma \leqslant 2$ the requirement $\Omega_{Y}(k, \tau) \ll 1$ always holds for $\mid k \tau \ll 1$ but, according to the general wisdom, the magnetogenesis constraints cannot be satisfied since the hypermagnetic power spectrum is still too violet;

- If $\gamma>2$ the hypermagnetic spectrum becomes even steeper while the hyperelectric spectrum diverges in the limit $k \tau \ll 1$ : in this case the bound $\Omega_{Y} \ll 1$ is not satisfied so that the whole class of models $\gamma>2$ must be excluded.

The only case not explicitly covered is $\gamma \rightarrow 1 / 2$ but in this case both the electric and the magnetic spectra are steeply increasing while the magnetic spectrum inherits a logarithmic correction $^{9}$ :

$$
\begin{aligned}
& P_{B}(k, \tau)=\frac{a^{4} H^{4}}{8 \pi}(-k \tau)^{5}\left|H_{0}^{(1)}(-k \tau)\right|^{2} \rightarrow \frac{a^{4} H^{4}}{2 \pi^{3}}|k \tau|^{5} \ln ^{2}|k \tau|, \\
& P_{E}(k, \tau)=\frac{a^{4} H^{4}}{8 \pi}(-k \tau)^{5}\left|H_{1}^{(1)}(-k \tau)\right|^{2} \rightarrow \frac{a^{4} H^{4}}{2 \pi^{3}}|k \tau|^{3} \\
& \Omega_{Y}(k, \tau)=\frac{1}{3 \pi^{3}}\left(\frac{H}{M_{P}}\right)^{2}|k \tau|^{3}\left[1+|k \tau|^{2} \ln ^{2}|k \tau|\right]
\end{aligned}
$$

Therefore, also in the case $\gamma \rightarrow 1 / 2$ the spectral energy density is subcritical when the typical wavelengths are larger than the effective horizon.

If we consider equation (3.13) at face value we must admit that for $\gamma \leqslant 2$ the critical density constraint is always satisfied since both the hyperelectric and the hypermagnetic power spectra are extremely minute; the relation among them can be easily deduced from equations (3.11) and (3.12) and it is given by:

$$
\begin{aligned}
& P_{B}(k, \tau)=(-k \tau)^{2} P_{E}(k, \tau), \quad \text { for } \gamma>1 / 2, \\
& P_{B}(k, \tau)=(-k \tau)^{4 \gamma} P_{E}(k, \tau), \quad \text { for } 0<\gamma<1 / 2 .
\end{aligned}
$$

In both cases the hypermagnetic field spectrum is smaller than its hyperelectric counterpart. This feature may also arise in other models but it is not problematic as long as the power spectra are both very small. There have been recently various suggestions attempting to include spectator (electric) fields in curved backgrounds [48, 49] with the purpose of estimating the Schwinger effect in de Sitter space-time. These estimates are typically done by assuming a constant and uniform electric field in de Sitter space and by also postulating a vector-like coupling to fermions. These three assumptions do not specifically hold in the present case since the fields obtained here are non-uniform and non-homogeneous, they are not constant in time and they have, in general, a chiral coupling to fermions.

It is interesting to note that the estimates of the Schwinger-like effects are highly non-generic in the sense that to have a sizable effect we need to concoct specific currents forbidding the dilution of the electric energy density by the expansion of the Universe. Indeed, the constancy and uniformity of the field configurations entering the estimate of the above effect is achieved by considering a class of appropriate currents and this physical situation is markedly different from the flat space-time case where the constancy and uniformity of the field imposes the

\footnotetext{
${ }^{9}$ When $\gamma \rightarrow 1 / 2$ the mode functions become $f_{k}(\tau)=N_{0} \sqrt{-k \tau} H_{0}^{(1)}(-k \tau) / \sqrt{2 k}$ and $g_{k}(\tau)=N_{0} \sqrt{k / 2}$ $\sqrt{-k \tau} H_{1}^{(1)}(-k \tau)$, where $N_{0}=\sqrt{\pi / 2} \mathrm{e}^{\mathrm{i} \pi / 4}$.
} 
absence of time-dependent currents. The idea of reference [48] (somehow questioned in reference [49]) is that to achieve a physical electric field that is constant ${ }^{10}$ one must actually require the presence of a comoving current so that the explicit form of the corresponding evolution equations will be:

$$
\nabla \times \vec{E}=0, \quad \nabla \times \vec{B}=0, \quad \vec{J}+\vec{E}^{\prime}=0, \quad \vec{B}^{\prime}=0 .
$$

If one would simply choose $\vec{E}=E_{0} \hat{n}$ (where $\hat{n}$ is a certain unit vector), $\vec{B}=0$ and $\vec{J}=0$ this would be the standard Schwinger ansatz but the rate of pair production in de Sitter space would be negligible since the physical fields will be suppressed as $\vec{E}^{\text {(phys) }}=E_{0} \hat{n} / a^{2}$. To have a nonnegligible effect the common wisdom is therefore to postulate an ad hoc current constructed in such a way that the resulting physical field is a space-time constant. This can be achieved by postulating a comoving field in the form $\vec{E}=a^{\lambda} E_{0} \hat{n}$ [49] implying that the corresponding current becomes, from equation (3.19), $\vec{J}=-\lambda a H \vec{E}$. If $\lambda \leqslant 2$ the corresponding physical field is less suppressed and it becomes constant in the limit $\lambda \rightarrow 2$. These spectator electric fields can be understood as the result of an effective conductivity $\sigma=-\lambda H a$ which is negative if the Universe expands (as we are discussing here). It has been argued that this approach may cause a violation of the second law of thermodynamics [49]. It is debatable if this kind of estimates are at all relevant for the situation described in this paper. Even assuming that the coupling of hypercharge fields is vector-like (which is not the case as discussed above), the effects of the current $\vec{J}=-\lambda a H \vec{E}$ are physically quite different from the ones associated with the spectra (3.17) and (3.18) which are fully inhomogeneous, time-dependent and obtained in the absence of any supporting current.

Having specified what is the real physical situation we can always try to see under which conditions the probability of pair creation per unit volume per unit time is under control in some averaged sense. Taking into account all the preceding caveats, and denoting with $\Gamma$ the rate of pair production per unit volume and per unit time a reasonable condition to consider seems to be:

$$
\frac{\Gamma}{H^{4}}=\frac{\alpha}{\pi^{2}}\left\langle E^{2}(\tau, \vec{x})\right\rangle<1, \quad \Rightarrow \frac{2}{\pi^{2} \lambda} \int_{0}^{k_{\max }} \frac{\mathrm{d} k}{k} P_{E}(k, \tau)<H^{4},
$$

where we recalled that, within the conventions spelled out in equation (2.17), $\alpha=e^{2} /(4 \pi)=$ $1 / \lambda$; we also assumed that the flat-space result holds (locally) over typical scales $\mathcal{O}\left(H^{-1}\right)$ during the quasi-de Sitter stage. Let us now consider, for simplicity, the case of equation (3.17) and see if and how the condition (3.20) is satisfied. In equation (3.20) $k_{\max }$ denotes the maximally amplified wavenumber which is given, in the present context $k_{\max }=\mathcal{O}\left(1 / \tau_{1}\right)$. By using the explicit form of the power spectrum given in equation (3.12) the condition (3.20) implies

$$
\frac{e_{1}^{2} 2^{2 \gamma-3} \Gamma^{2}(\gamma+1 / 2) \zeta^{4-2 \gamma}}{(4-2 \gamma) \pi^{5}}\left(\frac{a_{1}}{a}\right)^{-4 \gamma} \ll 1, \quad 0<\gamma<2,
$$

where $\zeta$ is a numerical factor $\mathcal{O}(1)$ (we parametrized $k_{\max }=\zeta / \tau_{1}$ by following the observations discussed after equation (2.40)). The condition equation (3.21) is always verified for $\tau<-\tau_{1}$ since in the wanted range of $\gamma$ we have that $\left(a_{1} / a\right)^{-4 \gamma}=\left(-\tau / \tau_{1}\right)^{-4 \gamma} \ll 1$. This suggests that the condition (3.21) is always satisfied except, probably, at the very end of inflation. This is, however, not a problem of the physics but just a problem of the accuracy in the estimate of the inflationary spectrum around $k_{\max }$. Even in the worse situation the ideas conveyed

\footnotetext{
${ }^{10}$ It turns out to be useful, in this respect, the distinction between physical and comoving fields given in equation (2.12) The only caveat is that, in standard estimates of the Schwinger effect, the gauge coupling does not evolve.
} 
in this paper come do not contradict the spirit of the bound (3.21): since the gauge coupling at the end of inflation is effectively a tuneable parameter, it is sufficient to reduce $e_{1}^{2}$ by one order of magnitude to safely satisfy the conditions (3.20) and (3.21) also at the end of inflation. All in all equations (3.19)-(3.21) suggest that the constraints coming from pair production are therefore not essential in the present context even if an improved understanding of the whole problem would be desirable (see e.g. [49]) and discussions therein).

In summary when the gauge coupling increases during a quasi-de Sitter stage of expansion the spectral energy density is subcritical for $0<\gamma \leqslant 2$ and overcritical for $\gamma>2$ so that the latter range is excluded while the former is still viable. Since the hypermagnetic spectrum is rather steep (i.e. violet) when $\gamma=2$ the conventional wisdom is that it will also be minute at the galactic scale after the gauge coupling flattens out. This swift conclusion is only true provided the hypermagnetic magnetic power spectrum at the end of inflation is not modified for $\tau \geqslant-\tau_{1}$. In section 4 the gauge power spectra will be explicitly computed in the regime where the gauge coupling flattens out (i.e. for $\tau \geqslant-\tau_{1}$ ) and it will be shown that the latetime hypermagnetic spectrum does not coincide with the hypermagnetic spectrum at the end of inflation when the gauge coupling increases.

\subsection{Decreasing gauge coupling}

When $\sqrt{\lambda}$ evolves as in equation (2.41) (see also figure 2) the correctly normalized solution of equations (2.20) and (2.21) are now given by

$$
f_{k}(\tau)=\frac{N_{\widetilde{\mu}}}{\sqrt{2 k}} \sqrt{-k \tau} H_{\widetilde{\mu}}^{(1)}(-k \tau), \quad g_{k}(\tau)=N_{\widetilde{\mu}} \sqrt{\frac{k}{2}} \sqrt{-k \tau} H_{\widetilde{\mu}-1}^{(1)}(-k \tau),
$$

where

$$
\widetilde{\mu}=\widetilde{\gamma}+\frac{1}{2}, \quad N_{\widetilde{\mu}}=\sqrt{\frac{\pi}{2}} \mathrm{e}^{\mathrm{i} \pi(2 \widetilde{\mu}+1) / 4} .
$$

Inserting equations (3.22) and (3.23) into equations (2.25) and (2.26) the comoving power spectra are:

$$
\begin{aligned}
& \widetilde{P}_{B}(k, \tau)=\frac{a^{4} H^{4}}{8 \pi}(-k \tau)^{5}\left|H_{\widetilde{\mu}}^{(1)}(-k \tau)\right|^{2} \rightarrow a^{4} H^{4} D(\widetilde{\mu})|k \tau|^{5-2 \widetilde{\mu}}, \\
& \widetilde{P}_{E}(k, \tau)=\frac{a^{4} H^{4}}{8 \pi}(-k \tau)^{5}\left|H_{\widetilde{\mu}-1}^{(1)}(-k \tau)\right|^{2} \rightarrow a^{4} H^{4} D(|\widetilde{\mu}-1|)|k \tau|^{5-2|\widetilde{\mu}-1|},
\end{aligned}
$$

where the function $D(x)$ has been already defined in equation (3.9) for a generic argument $x$. To stress that the power spectra (3.24) and (3.25) correspond to the case of decreasing gauge coupling a tilde has been added on top of each expression. Since, according to equation (3.23), $\widetilde{\mu}=\widetilde{\gamma}+1 / 2$, equations (3.24) and (3.25) are directly expressible in terms of $\widetilde{\gamma}$ and the result is:

$$
\begin{aligned}
& \widetilde{P}_{B}(k, \tau)=a^{4} H^{4} D(\widetilde{\gamma}+1 / 2)|k \tau|^{4-2 \widetilde{\gamma}}, \\
& \widetilde{P}_{E}(k, \tau)=a^{4} H^{4} D(|\widetilde{\gamma}-1 / 2|)|k \tau|^{5-|2 \widetilde{\gamma}-1|} .
\end{aligned}
$$

Equations (3.26) and (3.27) will now be inserted into equation (2.32); the explicit expression of the spectral energy density is:

$$
\widetilde{\Omega}_{Y}(k, \tau)=\frac{2}{3}\left(\frac{H}{M_{P}}\right)^{2}\left[D_{B}(\widetilde{\gamma}+1 / 2)|k \tau|^{4-2 \widetilde{\gamma}}+D_{E}(|\widetilde{\gamma}-1 / 2|)|k \tau|^{5-|2 \widetilde{\gamma}-1|}\right],
$$


where in analogy with equation (3.13) the subscripts $E$ and $B$ have been added to the function $D(x)$ with the purpose of reminding that the origin of the corresponding terms. As in the case of equation (3.13) the spectral energy density of equation (3.28) should always be subcritical (i.e. $\widetilde{\Omega}_{Y}(k, \tau) \ll 1$ for $\tau \leqslant-\tau_{1}$ and $\left.|k \tau| \ll 1\right)$. In this respect the following three observations are in order:

- When $\widetilde{\gamma}=2$ the hypermagnetic spectrum is flat while the hyperelectric spectrum is violet and it goes as $\widetilde{P}_{E}(k, \tau) \propto|k \tau|^{2}$; the spectral energy density is always subcritical for $|k \tau| \ll 1$;

- If $\widetilde{\gamma}=3$ the hyperelectric spectrum is flat however the hypermagnetic power spectrum diverges as $|k \tau|^{-2}$ in the limit $|k \tau| \ll 1$ but, in this case, the spectral energy density constraint is not satisfied;

- In the $0<\widetilde{\gamma}<1 / 2$ the power spectra are both violet; all in all we conclude that the bound (3.28) is satisfied for $0<\widetilde{\gamma} \leqslant 2$.

The case $\widetilde{\gamma} \rightarrow 1 / 2$ is not explicitly covered by equation (3.28) so that, in this limit, we have ${ }^{11}$

$$
\begin{aligned}
& \widetilde{P}_{B}(k, \tau)=\frac{a^{4} H^{4}}{8 \pi}(-k \tau)^{5}\left|H_{1}^{(1)}(-k \tau)\right|^{2} \rightarrow \frac{a^{4} H^{4}}{2 \pi^{3}}|k \tau|^{3}, \\
& \widetilde{P}_{E}(k, \tau)=\frac{a^{4} H^{4}}{8 \pi}(-k \tau)^{5}\left|H_{0}^{(1)}(-k \tau)\right|^{2} \rightarrow \frac{a^{4} H^{4}}{2 \pi^{3}}|k \tau|^{5} \ln ^{2}|k \tau|, \\
& \widetilde{\Omega}_{Y}(k, \tau)=\frac{1}{3 \pi^{3}}\left(\frac{H}{M_{P}}\right)^{2}|k \tau|^{3}\left[1+|k \tau|^{2} \ln ^{2}|k \tau|\right] .
\end{aligned}
$$

\subsection{Duality and gauge power spectra during inflation}

A flat hypermagnetic spectrum is only consistent with the critical density bound provided $\widetilde{\gamma}=2$ (i.e. only when the gauge coupling is decreasing). Conversely a flat hyperelectric spectrum is only viable when the gauge coupling increases and $\gamma=2$. Overall the only intervals where the critical density constraint is satisfied are given by $0 \leqslant \gamma \leqslant 2$ and $0 \leqslant \widetilde{\gamma} \leqslant 2$. These results follow in fact from the duality symmetry of equations (2.16) and (2.29). For the gauge spectra during inflation the duality symmetry implies, in general:

$$
\sqrt{\lambda} \rightarrow \frac{1}{\sqrt{\lambda}}, \quad P_{B}(k, \tau) \rightarrow \tilde{P}_{E}(k, \tau), \quad P_{E}(k, \tau) \rightarrow \tilde{P}_{B}(k, \tau) .
$$

If now equations (3.11) and (3.12) are compared with equations (3.26) and (3.27) the spectra with increasing and decreasing gauge coupling during inflation are explicitly related by the following transformation:

$$
\gamma \rightarrow \widetilde{\gamma}, \quad P_{B}(k, \tau) \rightarrow \widetilde{P}_{E}(k, \tau), \quad P_{E}(k, \tau) \rightarrow \widetilde{P}_{B}(k, \tau)
$$

Equation (3.33) has actually the same content of equation (3.32) since, according to the explicit parametrizations of equations (2.33) and (2.41), the expression of $\sqrt{\lambda}$ is actually inverted when $\chi \rightarrow \widetilde{\gamma}$. Thanks equation (3.33) the spectral energy density is left invariant [i.e. $\Omega_{Y}(k, \tau) \rightarrow$ $\left.\Omega_{Y}(k, \tau)\right]$ since the hyperelectric and the hypermagnetic power spectra are interchanged. All in all the standard lore stipulates that the only phenomenologically relevant case is the one $\widetilde{\gamma}=2$

\footnotetext{
${ }^{11}$ When $\widetilde{\gamma}=1 / 2$ the solution of equations (2.20) and (2.21) is given by $f_{k}(\tau)=N_{1} \sqrt{-k \tau} H_{1}^{(1)}(-k \tau) / \sqrt{2 k}$ and by $g_{k}(\tau)=-N_{1} \sqrt{\frac{k}{2}} \sqrt{-k \tau} H_{0}^{(1)}(-k \tau)$.
} 
since the other cases lead to an hypermagnetic spectrum that is either too steep or anyway inconsistent with the critical density bound. As already mentioned, this statement assumes that the gauge spectra are unmodified when coupling flattens out and the relevant wavelengths are still larger than the effective horizon.

\section{Post-inflationary gauge spectra and continuity}

When the gauge coupling flattens out as illustrated in figures 1 and 2 the background geometry enters a stage of decelerated expansion after the end of the inflationary phase. The postinflationary gauge fields are determined by the continuous evolution of the corresponding mode functions whose late-time behavior follows from the elements of an appropriate transition matrix mixing together the hyperelectric and the hypermagnetic mode functions at the end of inflation and leading to specific standing oscillations. These Sakharov phases are different for the $(\gamma, \delta)$ transition illustrated in figure 1 and in the case of the $(\widetilde{\gamma}, \widetilde{\delta})$ profile of figure 2.

\subsection{Increasing gauge coupling}

4.1.1. Mixing matrix for the $(\gamma, \delta)$ transition. The continuous parametrization of $\sqrt{\lambda}$ given in equations (2.33) and (2.34) implies that the late-time values of $f_{k}(\tau)$ and $g_{k}(\tau)$ for $\tau \geqslant-\tau_{1}$ are given by:

$$
\left(\begin{array}{c}
f_{k}(\tau) \\
g_{k}(\tau) / k
\end{array}\right)=\left(\begin{array}{ll}
A_{f f}\left(k, \tau, \tau_{1}\right) & A_{f g}\left(k, \tau, \tau_{1}\right) \\
A_{g f}\left(k, \tau, \tau_{1}\right) & A_{g g}\left(k, \tau, \tau_{1}\right)
\end{array}\right)\left(\begin{array}{c}
\bar{f}_{k} \\
\bar{g}_{k} / k
\end{array}\right),
$$

where $\bar{f}_{k}=f_{k}\left(-\tau_{1}\right)$ and $\bar{g}_{k}=g_{k}\left(-\tau_{1}\right)$ denote the values of the mode functions at end of the inflationary phase and the matrix elements at the right-hand side of equation (4.1) are determined from the continuity of the mode functions as described in appendix A:

$$
\begin{aligned}
& A_{f f}\left(k, \tau, \tau_{1}\right)=\frac{\pi}{2} \sqrt{q x_{1}} \sqrt{k y}\left[Y_{\nu-1}\left(q x_{1}\right) J_{\nu}(k y)-J_{\nu-1}\left(q x_{1}\right) Y_{\nu}(k y)\right], \\
& A_{f g}\left(k, \tau, \tau_{1}\right)=\frac{\pi}{2} \sqrt{q x_{1}} \sqrt{k y}\left[J_{\nu}\left(q x_{1}\right) Y_{\nu}(k y)-Y_{\nu}\left(q x_{1}\right) J_{\nu}(k y)\right], \\
& A_{g f}\left(k, \tau, \tau_{1}\right)=\frac{\pi}{2} \sqrt{q x_{1}} \sqrt{k y}\left[Y_{\nu-1}\left(q x_{1}\right) J_{\nu-1}(k y)-J_{\nu-1}\left(q x_{1}\right) Y_{\nu-1}(k y)\right], \\
& A_{g g}\left(k, \tau, \tau_{1}\right)=\frac{\pi}{2} \sqrt{q x_{1}} \sqrt{k y}\left[J_{\nu}\left(q x_{1}\right) Y_{\nu-1}(k y)-Y_{\nu}\left(q x_{1}\right) J_{\nu-1}(k y)\right] .
\end{aligned}
$$

Since the inflationary mode functions $\bar{f}_{k}$ and $\bar{g}_{k}$ obey the Wronskian normalization of equation (2.21), also $f_{k}(\tau)$ and $g_{k}(\tau)$ must obey the same condition for $\tau \geqslant \tau_{1}$ and this happens provided $^{12}$ :

$$
A_{f f}\left(k, \tau, \tau_{1}\right) A_{g g}\left(k, \tau, \tau_{1}\right)-A_{f g}\left(k, \tau, \tau_{1}\right) A_{g f}\left(k, \tau, \tau_{1}\right)=1 .
$$

The arguments of the Bessel functions appearing in (4.2) depend on $q x_{1}$ and $k y$ while the corresponding indices depend on $\delta$; the explicit expressions of $q, y$ and $\nu$ are:

$$
q(\delta, \gamma)=\frac{\delta}{\gamma}, \quad y(\tau, \delta, \gamma)=\tau+\tau_{1}[1+q(\delta, \gamma)], \quad \nu(\delta)=\delta+1 / 2
$$

\footnotetext{
${ }^{12}$ The validity of this condition can also be verified by plugging the explicit matrix elements of equation (4.2) into (4.3) and by using the standard recurrence relations involving the Bessel functions and their Wronskians [42, 43].
} 
Equation (4.4) shows that, within the present notations, $y\left(-\tau_{1}\right)=q \tau_{1}$ which also implies (by definition of $\left.x_{1}\right)$ that $k y\left(-\tau_{1}\right)=q k \tau_{1}=q x_{1}$. Consequently, as expected from the continuity of the mode functions,

$$
A_{f g}\left(k,-\tau_{1}, \tau_{1}\right)=A_{g f}\left(k,-\tau_{1}, \tau_{1}\right)=0, \quad A_{f f}\left(k,-\tau_{1}, \tau_{1}\right)=A_{g g}\left(k,-\tau_{1}, \tau_{1}\right)=1 .
$$

Since all the expression entering equation (4.2) ultimately depend on the dimensionless variables $x=k \tau, x_{1}=k \tau_{1}$ and $\nu$, the matrix appearing in equation (4.1) is in fact a function of $\delta$, $x$ and $x_{1}$ for any fixed value of $\gamma$ :

$$
\mathcal{M}\left(\delta, x, x_{1}\right)=\left(\begin{array}{ll}
A_{f f}\left(\delta, x, x_{1}\right) & A_{f g}\left(\delta, x, x_{1}\right) \\
A_{g f}\left(\delta, x, x_{1}\right) & A_{g g}\left(\delta, x, x_{1}\right)
\end{array}\right) .
$$

We stress that the variable $x_{1}=k \tau_{1} \leqslant 1$ measures $k$ in units of the maximal wavenumber of the spectrum (i.e. $\left.1 / \tau_{1}=a_{1} H_{1}\right)$ and this is why it cannot be larger than $\mathcal{O}(1)$.

4.1.2. General form of the power spectra. When the mode functions $f_{k}(\tau)$ and $g_{k}(\tau)$ are deduced from equation (4.1) the gauge power spectra of equations (2.25) and (2.26) become:

$$
\begin{aligned}
& P_{B}(k, \tau)=\frac{k^{5}}{2 \pi^{2}}\left|A_{f f} \bar{f}_{k}+A_{f g} \frac{\bar{g}_{k}}{k}\right|^{2}, \\
& P_{E}(k, \tau)=\frac{k^{3}}{2 \pi^{2}}\left|k A_{g f} \bar{f}_{k}+A_{g g} \bar{g}_{k}\right|^{2} .
\end{aligned}
$$

One of the two terms inside each of the squared moduli appearing in equations (4.7) and (4.8) will be alternatively dominant. For the sake of concreteness we shall now verify that the first term inside the squared modulus of equation (4.7) dominates against the second:

$$
\left|A_{f g}\left(\delta, x, x_{1}\right) \frac{\bar{g}_{k}}{k}\right| \gg\left|A_{f f}\left(\delta, x, x_{1}\right) \bar{f}_{k}\right| .
$$

Since all the variables have been explicitly defined, the validity of the condition (4.9) could be investigated numerically ${ }^{13}$. For a more general proof of equation (4.9) it is sufficient to consider equation (4.9) in the limit $x_{1}<1$ (which is always verified for all the amplified modes of the spectrum) together with the subsidiary condition that $0 \leqslant \delta \ll \gamma$. An equivalent form of equation (4.9) and the result is:

$$
\begin{aligned}
& \left|J_{\nu}\left(q x_{1}\right) Y_{\nu}(k y)-Y_{\nu}\left(q x_{1}\right) J_{\nu}(k y)\right| \\
& \quad \gg\left|\frac{H_{|\gamma-1 / 2|}^{(1)}\left(x_{1}\right)}{H_{\gamma+1 / 2}^{(1)}\left(x_{1}\right)}\right|\left|Y_{\nu-1}\left(q x_{1}\right) J_{\nu}(k y)-J_{\nu-1}\left(q x_{1}\right) Y_{\nu}(k y)\right| .
\end{aligned}
$$

If $x_{1} \ll x \ll 1$ the arguments of the Bessel and Hankel functions appearing in equation (4.10) are all smaller than 1. It then follows that, in this limit (i.e. $x_{1} / x=\tau_{1} / \tau \ll 1$ ), the inequalities (4.9) and (4.10) are verified provided:

$$
\left(\frac{q x_{1}}{2}\right)^{-2 \delta} \gg \frac{\Gamma(1 / 2-\delta) \Gamma(|\gamma-1 / 2|)}{\Gamma(1 / 2+\delta) \Gamma(\gamma+1 / 2)}\left(\frac{x_{1}}{2}\right)^{\gamma+1 / 2-|\gamma-1 / 2|},
$$

\footnotetext{
${ }^{13}$ This analysis has been performed by fixing $\gamma$ to a reference value and by scanning the relative weight of the two terms of equation (4.9) for different values of $\delta \ll \gamma$. For the sake of conciseness this numerical analysis will not be explicitly discussed.
} 
where we used the explicit result of equation (A.9). When $\gamma>1 / 2$ the condition (4.11) implies, up to irrelevant numerical factors, that $\left(q x_{1} / 2\right)^{-2 \delta}>\left(x_{1} / 2\right)$ which is always verified ${ }^{14}$ as long as $x_{1}<1$ and $\delta \geqslant 0$. Similarly, if $0<\gamma<1 / 2$, equation (4.11) implies $\left(q x_{1} / 2\right)^{-2 \delta}>\left(x_{1} / 2\right)^{\gamma}$ which is also verified in the physical range of the parameters.

So far we demonstrated that equation (4.9) holds in the range $x_{1} \ll x \ll 1$. When $x_{1} \ll 1$ and $x \gg 1$ the functions whose argument coincides with $k y \simeq x \gg 1$ can be always represented as ${ }^{15}$ :

$$
J_{\nu}(k y)=M_{\nu} \cos \theta_{\nu}, \quad Y_{\nu}(k y)=M_{\nu} \sin \theta_{\nu}
$$

where, for $x \gg 1, \theta_{\nu}(x) \rightarrow x$ while $M_{\nu}(x) \rightarrow \sqrt{2 / \pi} x^{-1 / 2}\left[1+\mathcal{O}\left(x^{-2}\right)\right]$; this is the so-called modulus-phase approximation for the Bessel functions [42, 43]. Inserting equation (4.12) into equation (4.10) and expanding the remaining functions for $x_{1} \ll 1$ it then follows that the inequality (4.9) is satisfied. Consequently, thanks to equation (4.9) the comoving spectrum of equation (4.7) is:

$$
P_{B}(k, \tau)=\frac{k^{5}}{2 \pi^{2}}\left|A_{f g}\left(\delta, x_{1}, x\right) \frac{\bar{g}_{k}}{k}\right|^{2} .
$$

The results of equations (4.9)-(4.13) show that the hyperelectric field at the end of inflation determines the late-time hypermagnetic field for $\tau \gg-\tau_{1}$. This is of course not a general truism but it happens provided the gauge coupling first increases during inflation and then flattens out in the radiation-dominated epoch. For the hyperelectric spectrum of equation (4.8) the inequality of equation (4.9) is in fact replaced by the following condition

$$
\left|A_{g g}\left(\delta, x, x_{1}\right) \bar{g}_{k}\right| \gg\left|A_{g f}\left(\delta, x, x_{1}\right) k \bar{f}_{k}\right|,
$$

which can be verified explicitly by using the same strategy illustrated in the case of equation (4.9); for the sake of conciseness these details will not be explicitly discussed. Therefore, thanks to equation (4.14), the late-time expression of the comoving hyperelectric spectrum is:

$$
P_{E}(k, \tau)=\frac{k^{3}}{2 \pi^{2}}\left|A_{g f}\left(\delta, x_{1} x\right) \bar{g}_{k}\right|^{2}
$$

Equation (4.15) mirrors the result of equation (4.13) and it shows that the hyperelectric power spectrum for $\tau \gg-\tau_{1}$ is determined by the hyperelectric power spectrum at $\tau=-\tau_{1}$. As we shall see in a moment when the gauge coupling decreases the dual result will hold.

4.1.3. Explicit expressions of the power spectra in various regimes. To obtain a more explicit form of the gauge power spectra in the decelerated stage of expansion the matrix elements of equation (4.2) can be expanded in powers of $x_{1} \ll 1$. The strategy will be to fix $k y$ and expand the various terms in powers of $x_{1}$ with the subsidiary conditions $0 \leqslant \delta \ll 1 / 2$. With

\footnotetext{
${ }^{14}$ In the limit $\delta \rightarrow 0$, since $q=\delta / \gamma$, the condition $\left(q x_{1} / 2\right)^{-2 \delta} \gg\left(x_{1} / 2\right)$ is also verified as long as $x_{1}<1$. Since the gauge coupling freezes (either partially or totally) for $\tau>-\tau_{1}$ the physical situation discussed here corresponds to a range of parameters where $0 \leqslant \delta \ll 1 / 2$ which also implies $0 \leqslant \delta \ll \gamma$; these two conditions will be used interchangeably.

${ }^{15}$ Recall, in this respect, equation (4.4). Even if the value of $x$ can be either smaller or larger than 1 , as soon as $x=k \tau=\mathcal{O}(1)$ the conductivity cannot be neglected and this situation will be more specifically discussed in section 5 . For the moment we shall just consider the case $x \gg 1$ as a mere mathematical limit.
} 
these specifications equation (4.2) implies ${ }^{16}$ :

$$
\begin{aligned}
& A_{f f}\left(\delta, x_{1}, x\right)=\left(\frac{q x_{1}}{2}\right)^{\delta}\left[\sqrt{\frac{x}{2}} \Gamma(1 / 2-\delta) J_{-\delta-1 / 2}(x)+\mathcal{O}\left(x_{1}\right)\right]+\mathcal{O}\left[\left(q x_{1}\right)^{1-\delta}\right], \\
& A_{f g}\left(\delta, x_{1}, x\right)=\left(\frac{q x_{1}}{2}\right)^{-\delta}\left[\sqrt{\frac{x}{2}} \Gamma(1 / 2+\delta) J_{\delta+1 / 2}(x)+\mathcal{O}\left(x_{1}\right)\right]+\mathcal{O}\left[\left(q x_{1}\right)^{1+\delta}\right], \\
& A_{g f}\left(\delta, x_{1}, x\right)=\left(\frac{q x_{1}}{2}\right)^{\delta}\left[-\sqrt{\frac{x}{2}} \Gamma(1 / 2-\delta) J_{1 / 2-\delta}(x)+\mathcal{O}\left(x_{1}\right)\right]+\mathcal{O}\left[\left(q x_{1}\right)^{1-\delta}\right], \\
& A_{g g}\left(\delta, x_{1}, x\right)=\left(\frac{q x_{1}}{2}\right)^{-\delta}\left[\sqrt{\frac{x}{2}} \Gamma(1 / 2+\delta) J_{\delta-1 / 2}(x)+\mathcal{O}\left(x_{1}\right)\right]+\mathcal{O}\left[\left(q x_{1}\right)^{1+\delta}\right] .
\end{aligned}
$$

Thanks to the results of equations (4.16) and (4.17) the inequality equation (4.9) can be again verified in a different manner. Furthermore, inserting equation (4.17) into equation (4.13) and recalling the expressions for $\bar{f}_{k}$ and $\bar{g}_{k}$ in the case of increasing coupling (see e.g. equation (A.8)) the hypermagnetic power spectrum becomes:

$$
\begin{aligned}
& P_{B}(k, \tau)=a_{1}^{4} H_{1}^{4} D(\gamma+1 / 2)\left(\frac{k}{a_{1} H_{1}}\right)^{4-2 \gamma-2 \delta} F_{B}(k \tau, \delta), \\
& F_{B}(x, \delta)=\left(\frac{q}{2}\right)^{-2 \delta}\left(\frac{x}{2}\right) \Gamma^{2}(\delta+1 / 2) J_{\delta+1 / 2}^{2}(x) .
\end{aligned}
$$

Similarly, from equations (4.15) and (4.19) the hyperelectric spectrum turns out to be

$$
\begin{aligned}
& P_{E}(k, \tau)=a_{1}^{4} H_{1}^{4} D(\gamma+1 / 2)\left(\frac{k}{a_{1} H_{1}}\right)^{4-2 \gamma-2 \delta} F_{E}(k \tau, \delta), \\
& F_{E}(x, \delta)=\left(\frac{q}{2}\right)^{-2 \delta}\left(\frac{x}{2}\right) \Gamma^{2}(\delta+1 / 2) J_{\delta-1 / 2}^{2}(x) .
\end{aligned}
$$

The results of equations (4.20) and (4.21) only assume $x_{1}<1$ and $0 \leqslant \delta \ll \gamma$ and can be evaluated either for $k \tau \ll 1$ or for $k \tau \gg 1$. As long as $k \tau \ll 1$ it is enough to recall that $J_{\alpha}(z) \simeq$ $(z / 2)^{\alpha} / \Gamma(\alpha+1)[42,43]$; in the opposite regime (i.e. $k \tau \gg 1$ ) equation (4.12) provides instead the valid approximation scheme $\mathrm{e}^{17}$.

Another interesting limit is the sudden approximation which is not well defined a priori but only as the $\delta \rightarrow 0$ limit; in this case $x$ and $x_{1}$ are kept fixed and the matrix elements of equation (4.2) assume a rather simple form implying:

$$
\begin{aligned}
& P_{B}(k, \tau)=\frac{k^{5}}{2 \pi^{2}}\left|\cos \left(x+x_{1}\right) \bar{f}_{k}+\sin \left(x+x_{1}\right) \frac{\bar{g}_{k}}{k}\right|^{2}, \\
& P_{E}(k, \tau)=\frac{k^{3}}{2 \pi^{2}}\left|-k \sin \left(x+x_{1}\right) \bar{f}_{k}+\cos \left(x+x_{1}\right) \bar{g}_{k}\right|^{2} .
\end{aligned}
$$

\footnotetext{
${ }^{16}$ According to equation (4.4), $k y=x+x_{1}(q+1)$ and, to lowest order in $x_{1}$, we have that $k y=x+\mathcal{O}\left(x_{1}\right)$.

${ }^{17}$ Equations (4.20) and (4.21) hold for any value of $k \tau$; however, as we shall argue in section 5, for $\tau>\tau_{k} \sim 1 / k$ the power spectra will be modified by the finite value of the conductivity.
} 
Using equation (A.8) the gauge power spectra will be:

$$
\begin{aligned}
& P_{B}(k, \tau)=a_{1}^{4} H_{1}^{4} D(\gamma+1 / 2)\left(\frac{k}{a_{1} H_{1}}\right)^{4-2 \gamma} \sin ^{2} k \tau, \\
& P_{E}(k, \tau)=a_{1}^{4} H_{1}^{4} D(\gamma+1 / 2)\left(\frac{k}{a_{1} H_{1}}\right)^{4-2 \gamma} \cos ^{2} k \tau .
\end{aligned}
$$

The same results of equations (4.24) and (4.25) follow immediately from equations (4.20) and (4.21) by recalling that $q^{-\delta}=(\delta / \gamma)^{-\delta} \rightarrow 1$ in the limit $\delta \rightarrow 0$. All in all, in the sudden approximation $x_{1}$ and $x$ are kept fixed while $\delta \rightarrow 0$; in the smooth limit $\delta$ may be very small (i.e. $\delta \ll 1$ ) but it is always different from zero. These two complementary approximations commute since it can be shown that:

$$
\lim _{\delta \rightarrow 0}\left[\lim _{x_{1} \ll x \ll 1} \mathcal{M}\left(\delta, x_{1}, x\right)\right]=\lim _{x_{1} \ll x \ll 1}\left[\lim _{\delta \rightarrow 0} \mathcal{M}\left(\delta, x_{1}, x\right)\right]=\left(\begin{array}{cc}
1 & x \\
-x & 1
\end{array}\right) .
$$

\subsection{Decreasing gauge coupling}

4.2.1. Matrix for the $(\widetilde{\gamma}, \widetilde{\delta})$ transition. When the gauge coupling decreases and the dynamics of the transition follows the timeline of figure 2 the analog of equation (4.1) can be written as:

$$
\left(\begin{array}{c}
f_{k}(\tau) \\
g_{k}(\tau) / k
\end{array}\right)=\left(\begin{array}{ll}
\widetilde{A}_{f f}\left(k, \tau, \tau_{1}\right) & \widetilde{A}_{f g}\left(k, \tau, \tau_{1}\right) \\
\widetilde{A}_{g f}\left(k, \tau, \tau_{1}\right) & \widetilde{A}_{g g}\left(k, \tau, \tau_{1}\right)
\end{array}\right)\left(\begin{array}{c}
\bar{f}_{k} \\
\bar{g}_{k} / k
\end{array}\right),
$$

where, as usual, $\bar{f}_{k}=f_{k}\left(-\tau_{1}\right)$ and $\bar{g}_{k}=g_{k}\left(-\tau_{1}\right)$ follow from equation (3.22) and their explicit expression can be found in equation (B.9). As usual we also added a tilde on top of the various matrix elements to stress that they are computed in the framework of the $(\widetilde{\gamma}, \widetilde{\delta})$ transition. Thanks to the Wronskian normalization (2.21) the analog of equation (4.3) is now:

$$
\widetilde{A}_{f f}\left(k, \tau, \tau_{1}\right) \widetilde{A}_{g g}\left(k, \tau, \tau_{1}\right)-\widetilde{A}_{f g}\left(k, \tau, \tau_{1}\right) \widetilde{A}_{g f}\left(k, \tau, \tau_{1}\right)=1 .
$$

The various entries of the transition matrix appearing in equation (4.27) are:

$$
\begin{aligned}
& \widetilde{A}_{f f}\left(k, \tau, \tau_{1}\right)=\frac{\pi}{2} \sqrt{q x_{1}} \sqrt{k y}\left[Y_{\widetilde{\nu}-1}\left(q x_{1}\right) J_{\widetilde{\nu}}(k y)-J_{\widetilde{\nu}-1}\left(q x_{1}\right) Y_{\widetilde{\nu}}(k y)\right], \\
& \widetilde{A}_{f g}\left(k, \tau, \tau_{1}\right)=\frac{\pi}{2} \sqrt{q x_{1}} \sqrt{k y}\left[J_{\widetilde{\nu}}\left(q x_{1}\right) Y_{\widetilde{\nu}}(k y)-Y_{\widetilde{\nu}}\left(q x_{1}\right) J_{\widetilde{\nu}}(k y)\right], \\
& \widetilde{A}_{g f}\left(k, \tau, \tau_{1}\right)=\frac{\pi}{2} \sqrt{q x_{1}} \sqrt{k y}\left[Y_{\widetilde{\nu}-1}\left(q x_{1}\right) J_{\widetilde{\nu}-1}(k y)-J_{\widetilde{\nu}-1}\left(q x_{1}\right) Y_{\widetilde{\nu}-1}(k y)\right], \\
& \widetilde{A}_{g g}\left(k, \tau, \tau_{1}\right)=\frac{\pi}{2} \sqrt{q x_{1}} \sqrt{k y}\left[J_{\widetilde{\nu}}\left(q x_{1}\right) Y_{\widetilde{\nu}-1}(k y)-Y_{\widetilde{\nu}}\left(q x_{1}\right) J_{\widetilde{\nu}-1}(k y)\right] .
\end{aligned}
$$

The matrix elements of equation (4.29) and the values of $\bar{f}_{k}$ and $\bar{g}_{k}$ are determined from the continuity of the mode functions as described in appendix B. The variables $y, q$ and $\widetilde{\nu}$ are now functions of $\widetilde{\delta}$ and $\widetilde{\gamma}$ in full analogy with equation (4.4):

$$
q(\widetilde{\delta}, \widetilde{\gamma})=\frac{\widetilde{\delta}}{\widetilde{\gamma}}, \quad y(\tau, \widetilde{\delta}, \widetilde{\gamma})=\tau+\tau_{1}[1+q(\widetilde{\delta}, \widetilde{\gamma})], \quad \widetilde{\nu}(\widetilde{\delta})=|\widetilde{\delta}-1 / 2|
$$


Note that equation (4.29) has the same form of equation (4.2); formally equation (4.29) can be obtained from equation (4.2) by replacing $\nu$ with $\widetilde{\nu}$. With the same notations of equation (4.6) we can define the matrix

$$
\widetilde{\mathcal{M}}\left(\widetilde{\delta}, x, x_{1}\right)=\left(\begin{array}{ll}
\widetilde{A}_{f f}\left(\widetilde{\delta}, x, x_{1}\right) & \widetilde{A}_{f g}\left(\widetilde{\delta}, x, x_{1}\right) \\
\widetilde{A}_{g f}\left(\widetilde{\delta}, x, x_{1}\right) & \widetilde{A}_{g g}\left(\widetilde{\delta}, x, x_{1}\right)
\end{array}\right) .
$$

Inserting the expressions of equation (4.27) into equations (2.25) and (2.26) the comoving power spectra for the $(\widetilde{\gamma}, \widetilde{\delta})$ transition are:

$$
\begin{aligned}
& \widetilde{P}_{B}(k, \tau)=\frac{k^{5}}{2 \pi^{2}}\left|\widetilde{A}_{f f} \bar{f}_{k}+\widetilde{A}_{f g} \frac{\bar{g}_{k}}{k}\right|^{2}, \\
& \widetilde{P}_{E}(k, \tau)=\frac{k^{3}}{2 \pi^{2}}\left|k \widetilde{A}_{g f} \bar{f}_{k}+\widetilde{A}_{g g} \bar{g}_{k}\right|^{2} .
\end{aligned}
$$

As already argued for the $(\gamma, \delta)$ transition, the terms appearing inside the squared moduli at the right-hand side of equations (4.32) and (4.33) are not of the same order but one of the two terms will be alternatively dominant. Owing to the specific form of equations (4.31), (4.29) and (B.9) the hierarchies between the different terms contributing to equations (4.32) and (4.33) turn out to be:

$$
\begin{aligned}
& \left|\widetilde{A}_{f f}\left(\widetilde{\delta}, x, x_{1}\right) \bar{f}_{k}\right| \gg\left|\widetilde{A}_{f g}\left(\widetilde{\delta}, x, x_{1}\right) \frac{\bar{g}_{k}}{k}\right|, \\
& \left|\widetilde{A}_{g f}\left(\widetilde{\delta}, x, x_{1}\right) \bar{f}_{k}\right| \gg\left|\widetilde{A}_{g g}\left(\widetilde{\delta}, x, x_{1}\right) \frac{\bar{g}_{k}}{k}\right| .
\end{aligned}
$$

The inequalities equations (4.34) and (4.35) imply that the late-time hypermagnetic power spectra are determined by the hypermagnetic power spectra at $\tau_{1}$ and they can be explicitly verified by following the same strategy illustrated in the case of the $(\gamma, \delta)$ transition. So for instance we can rewrite equation (4.34) as:

$$
\begin{aligned}
& \left|Y_{\widetilde{\nu}-1}\left(q x_{1}\right) J_{\widetilde{\nu}}(k y)-J_{\widetilde{\nu}-1}\left(q x_{1}\right) Y_{\widetilde{\nu}}(k y)\right| \\
& \quad \gg\left|\frac{H_{\widetilde{\mu}-1}^{(1)}\left(x_{1}\right)}{H_{\widetilde{\mu}}^{(1)}\left(x_{1}\right)}\right|\left|J_{\widetilde{\nu}}\left(q x_{1}\right) Y_{\widetilde{\nu}}(k y)-Y_{\widetilde{\nu}}\left(q x_{1}\right) J_{\widetilde{\nu}}(k y)\right| .
\end{aligned}
$$

If both sides of equation (4.36) are expanded for $x_{1} \ll x<1$ and with the subsidiary condition $0 \leqslant \widetilde{\delta} \ll \widetilde{\gamma}$ we obtain the following condition:

$$
\left(\frac{q x_{1}}{2}\right)^{2 \widetilde{\delta}} \gg \frac{\Gamma(3 / 2+\widetilde{\delta}) \Gamma(|\widetilde{\gamma}-1 / 2|)}{\Gamma(1 / 2-\widetilde{\delta}) \Gamma(\widetilde{\gamma}+1 / 2)}\left(\frac{x_{1}}{2}\right)^{\widetilde{\gamma}+1 / 2-|\widetilde{\gamma}-1 / 2|}
$$

When $\widetilde{\gamma}>1 / 2$ equation (4.37) implies $x_{1}^{2 \widetilde{\delta}}>x_{1}$ : this requirement is always verified for $x_{1}<1$ since, by assumption, $0 \leqslant \widetilde{\delta} \ll 1 / 2$. Similarly, in the range $\widetilde{\gamma}<1 / 2$, equation (4.37) demands $x_{1}^{2 \widetilde{\delta}}>x_{1}^{2 \widetilde{\gamma}}$ which is also true since $\widetilde{\delta} \ll \widetilde{\gamma}$. The same logic can be applied to equation (4.35) but this discussion will be omitted for the sake of conciseness. All in all equations (4.34) and (4.35) imply that the comoving power spectra become

$$
\widetilde{P}_{B}(k, \tau)=\frac{k^{5}}{2 \pi^{2}}\left|\widetilde{A}_{f f}\left(\widetilde{\delta}, x_{1}, x\right) \bar{f}_{k}\right|^{2}, \quad \widetilde{P}_{E}(k, \tau)=\frac{k^{3}}{2 \pi^{2}}\left|\widetilde{A}_{g f}\left(\widetilde{\delta}, x_{1}, x\right) k \bar{f}_{k}\right|^{2}
$$


4.2.2. Explicit forms of the power spectra in various limits. The spectra of equation (4.38) can be evaluated in various limits and, for this purpose, it is useful to expand equation (4.29) for $x_{1}$ with the subsidiary conditions $0 \leqslant \delta<1 / 2$. The result of this stem can be expressed as:

$$
\begin{aligned}
& \widetilde{A}_{f f}\left(\widetilde{\delta}, x_{1}, x\right)=\left(\frac{q x_{1}}{2}\right)^{-\widetilde{\delta}}\left[\sqrt{\frac{x}{2}} \Gamma(1 / 2+\widetilde{\delta}) J_{\widetilde{\delta}-1 / 2}(x)+\mathcal{O}\left(x_{1}\right)\right]+\mathcal{O}\left[\left(q x_{1}\right)^{1+\widetilde{\delta}}\right], \\
& \widetilde{A}_{f g}\left(\widetilde{\delta}, x_{1}, x\right)=\left(\frac{q x_{1}}{2}\right)^{\widetilde{\delta}}\left[\sqrt{\frac{x}{2}} \Gamma(1 / 2-\widetilde{\delta}) J_{-\widetilde{\delta}+1 / 2}(x)+\mathcal{O}\left(x_{1}\right)\right]+\mathcal{O}\left[\left(q x_{1}\right)^{1-\widetilde{\delta}}\right], \\
& \widetilde{A}_{g f}\left(\widetilde{\delta}, x_{1}, x\right)=\left(\frac{q x_{1}}{2}\right)^{-\widetilde{\delta}}\left[-\sqrt{\frac{x}{2}} \Gamma(1 / 2+\widetilde{\delta}) J_{1 / 2+\widetilde{\delta}}(x)+\mathcal{O}\left(x_{1}\right)\right]+\mathcal{O}\left[\left(q x_{1}\right)^{1+\widetilde{\delta}}\right], \\
& \widetilde{A}_{g g}\left(\widetilde{\delta}, x_{1}, x\right)=\left(\frac{q x_{1}}{2}\right)^{\widetilde{\delta}}\left[\sqrt{\frac{x}{2}} \Gamma(1 / 2-\widetilde{\delta}) J_{-\widetilde{\delta}-1 / 2}(x)+\mathcal{O}\left(x_{1}\right)\right]+\mathcal{O}\left[\left(q x_{1}\right)^{1-\widetilde{\delta}}\right] .
\end{aligned}
$$

Again using equations (4.39), (4.40) and (4.41), (4.42) the inequalities (4.34) and (4.35) are verified and the corresponding power spectra turn out to be:

$$
\begin{aligned}
& \widetilde{P}_{B}(k, \tau)=a_{1}^{4} H_{1}^{4} D(\widetilde{\gamma}+1 / 2)\left(\frac{k}{a_{1} H_{1}}\right)^{4-2 \widetilde{\gamma}-2 \widetilde{\delta}} \widetilde{F}_{B}(k \tau, \widetilde{\delta}), \\
& \widetilde{F}_{B}(x, \widetilde{\delta})=\left(\frac{q}{2}\right)^{-2 \widetilde{\delta}}\left(\frac{x}{2}\right) \Gamma^{2}(\delta+1 / 2) J_{\widetilde{\delta}-1 / 2}^{2}(x) .
\end{aligned}
$$

From equations (4.15) and (4.19) the hyperelectric spectrum is

$$
\begin{aligned}
& \widetilde{P}_{E}(k, \tau)=a_{1}^{4} H_{1}^{4} D(\widetilde{\gamma}+1 / 2)\left(\frac{k}{a_{1} H_{1}}\right)^{4-2 \widetilde{\gamma}-2 \widetilde{\delta}} \widetilde{F}_{E}(k \tau, \widetilde{\delta}), \\
& \widetilde{F}_{E}(x, \widetilde{\delta})=\left(\frac{q}{2}\right)^{-2 \widetilde{\delta}}\left(\frac{x}{2}\right) \Gamma^{2}(\widetilde{\delta}+1 / 2) J_{\widetilde{\delta}+1 / 2}^{2}(x) .
\end{aligned}
$$

Equations (4.43) and (4.44) can be evaluated in the sudden approximation by taking the limit $\widetilde{\delta} \rightarrow 0$ while $x$ and $x_{1}$ are kept fixed; the gauge power spectra are, in this case ${ }^{18}$

$$
\begin{aligned}
& \widetilde{P}_{B}(k, \tau)=a_{1}^{4} H_{1}^{4} D(\widetilde{\gamma}+1 / 2)\left(\frac{k}{a_{1} H_{1}}\right)^{4-2 \widetilde{\gamma}} \cos ^{2} k \tau, \\
& \widetilde{P}_{E}(k, \tau)=a_{1}^{4} H_{1}^{4} D(\widetilde{\gamma}+1 / 2)\left(\frac{k}{a_{1} H_{1}}\right)^{4-2 \widetilde{\gamma}} \sin ^{2} k \tau .
\end{aligned}
$$

By comparing equations (4.45) and (4.46) with the analog results given in equations (4.24) and (4.25) we note that the phases of the Sakharov oscillations are exchanged: while $P_{B}(k, \tau)$ oscillates like $\sin ^{2} k \tau$, the oscillations of $\widetilde{P}_{B}(k, \tau)$ go like $\cos ^{2} k \tau$. The opposite is true for the hyperelectric power spectra. The differences in the phases of oscillation are a particular consequence of duality which will now be analyzed in general terms.

\footnotetext{
${ }^{18}$ Note that the same results of equations (4.45) and (4.46) follow immediately from equations (4.43) and (4.44) by recalling that $q^{-\widetilde{\delta}}=(\widetilde{\delta} / \widetilde{\gamma})^{-\widetilde{\delta}} \rightarrow 1$ in the limit $\widetilde{\delta} \rightarrow 0$.
} 


\subsection{Duality and continuity of the power spectra after inflation}

During inflation duality relates the gauge power spectra in different dynamical situations (see equations (3.32) and (3.33)). When the gauge coupling flattens out the gauge power spectra are also related by duality which is equivalent to the following transformation:

$$
(\gamma, \delta) \rightarrow(\widetilde{\gamma}, \widetilde{\delta})
$$

The action of equation (4.47) transforms the elements of the transition matrix according to

$$
A_{f f} \rightarrow \widetilde{A}_{g g}, \quad A_{g g} \rightarrow \widetilde{A}_{f f}, \quad A_{f g} \rightarrow-\widetilde{A}_{g f}, \quad A_{g f} \rightarrow-\widetilde{A}_{f g} .
$$

Equation (4.48) follows from the expressions of the matrix elements reported in equations (4.2) and (4.29). For the sake of accuracy the results of equation (4.48) have been explicitly derived in appendix C. Moreover $\bar{f}_{k}$ and $\bar{g}_{k}$ will transform under duality as $\bar{f}_{k} \rightarrow \bar{g}_{k} / k$ and $\bar{g}_{k} \rightarrow-k \bar{f}_{k}$. Thanks to equations (2.29) and (4.48) the action of equation (4.47) transforms the gauge power spectra as:

$$
P_{B}(k, \tau) \rightarrow \widetilde{P}_{E}(k, \tau), \quad P_{E}(k, \tau) \rightarrow \widetilde{P}_{B}(k, \tau)
$$

Also the approximate expressions of the power spectra, if they are correct, must transform according to equation (4.49) since they must be consistent with duality; in particular:

- The approximate power spectrum $P_{B}(k, \tau)$ of equation (4.20) gives the approximate form of $\widetilde{P}_{E}$ (i.e. equation (4.44));

- The transformation (4.47) applied to equation (4.21) gives the approximate form of the hypermagnetic power spectrum in the dual description (i.e. $P_{E}(k, \tau) \rightarrow \widetilde{P}_{B}(k, \tau)$ );

- In the sudden approximation (when $\delta \rightarrow 0$ and $\widetilde{\delta} \rightarrow 0$ ) the gauge power spectra of equations (4.7), (4.8) and (4.32), (4.33) are related by duality.

All in all during inflation duality implies that when the gauge coupling increases the magnetic spectrum is never flat and if the gauge coupling is instead decreasing the electric spectrum is never flat. After inflation duality constrains the explicit forms of the gauge power spectra and the phases of the Sakharov oscillations. If the gauge coupling flattens out after a phase of increasing coupling the late-time gauge spectra are determined by the hyperelectric spectrum at the end of inflation. In the dual situation the gauge coupling freezes after a stage of decreasing coupling. Barring for the possible physical justifications of a strongly coupled phase at the beginning of inflation, the late-time gauge power spectra follow from the hypermagnetic spectrum at the end of inflation.

\section{Phenomenological considerations}

The hypercharge field projects on the electromagnetic fields through the cosine of the Weinberg angle (i.e. $\cos \theta_{\mathrm{W}}$ ) so that the late-time gauge power spectra could be compared both with the magnetogenesis requirements and with other phenomenological constraints. In this section we adopt the standard notations commonly employed in the context of the concordance paradigm. So for instance $\Omega_{R 0}$ denotes present critical fraction of relativistic particles, $\Omega_{M 0}$ is total critical fraction of massive species, $r_{\mathrm{T}}$ defines the ratio between the tensor and scalar power spectra at the pivot scale $k_{p}=0.002 \mathrm{Mpc}^{-1}$ and so on and so forth. The present value of the scale factor will be normalized to 1 (i.e. $a_{0}=1$ ) and, thanks to this widely used convention, the physical and the comoving frequencies coincide at the present time. The present section is devoted to phenomenological implications; in particular the following topics will be specifically treated; 
- In subsection 5.1 a brief summary of the typical scales of the problem has been provided in a phenomenological perspective;

- In subsection 5.2 some simplified estimates of the physical spectra before reentry have been discussed;

- Subsection 5.3 is devoted to the physical spectra after reentry;

- In subsection 5.4 we give a specific discussion of the magnetogenesis constraints in a self-contained perspective;

- The dependence the parameter space of the model has been charted in the subsection 5.5;

- The subsection 5.6 has been devoted to the effects of a post-inflationary phase preceding the radiation epoch;

- A final assessment of the obtained results has been presented in the subsection 5.7.

\subsection{Scales of the problem}

Defining with $\tau_{k}=1 / k$ the reentry time of a generic wavelength, the ratio between $\tau_{k}$ and the time of matter-radiation equality $\tau_{\text {eq }}$ is:

$$
\frac{\tau_{k}}{\tau_{\mathrm{eq}}}=1.06 \times 10^{-2}\left(\frac{h_{0}^{2} \Omega_{M 0}}{0.1386}\right)\left(\frac{h_{0}^{2} \Omega_{R 0}}{4.15 \times 10^{-5}}\right)^{-1 / 2}\left(\frac{k}{\mathrm{Mpc}^{-1}}\right)^{-1}
$$

since $\left(\tau_{k} / \tau_{\text {eq }}\right)=\sqrt{2}\left(H_{0} / k\right) \Omega_{M 0} / \Omega_{R 0}$ and $H_{0}=100 h_{0}(\mathrm{kmMpc})^{-1} \mathrm{~Hz}$ denotes the present value of the Hubble rate. Consequently the relevant wavenumbers for magnetogenesis considerations [i.e. $k=\mathcal{O}\left(\mathrm{Mpc}^{-1}\right)$ ] reentered prior to matter radiation equality.

After the gauge coupling flattens out the amplitude of the comoving power spectra is $\mathcal{O}\left(H_{1}^{4}\right)$ (see, for instance, equations (4.20), (4.21) and (4.43), (4.44)). The inflationary Hubble rate $H_{1}$ is related to the Planck scale as ${ }^{19}$ :

$$
\frac{H_{1}}{M_{P}}=\sqrt{\pi \epsilon \mathcal{A}_{\mathcal{R}}}=2.17 \times 10^{-6}\left(\frac{r_{\mathrm{T}}}{0.01}\right)^{1 / 2}\left(\frac{\mathcal{A}_{\mathcal{R}}}{2.41 \times 10^{-9}}\right)^{1 / 2}
$$

where $\mathcal{A}_{\mathcal{R}}$ denotes the amplitude of the scalar power spectrum at the pivot scale $k_{p}=0.002 \mathrm{Mpc}^{-1}$. Thanks to equation (5.2) it is then possible to express $H_{1}^{4}$ in units of $\mathrm{nG}^{2}$ by recalling that $M_{P}^{4}=4.62 \times 10^{132} \mathrm{nG}^{2}$. The scale $H_{1}$ also enters the explicit expression of $x_{1}=k \tau_{1}=k /\left(a_{1} H_{1}\right)$ :

$$
\frac{k}{a_{1} H_{1}}=\frac{k}{a_{0} H_{0}}\left(\frac{a_{0} H_{0}}{a_{1} H_{1}}\right)=\frac{k}{H_{0}} \zeta_{r}^{1 / 2-\alpha}\left(2 \pi \epsilon \Omega_{R 0} \mathcal{A}_{\mathcal{R}}\right)^{-1 / 4} \sqrt{\frac{H_{0}}{M_{P}}}
$$

where the first equality is just true by definition while the second one is the explicit estimate. As already mentioned, in equation (5.3) $\Omega_{R 0}$ is the (present) critical fraction of massless species (in the concordance paradigm $h_{0}^{2} \Omega_{R 0}=4.15 \times 10^{-5}$ ); note that $H_{0}$ is usefully expressed in Planck units as $\left(H_{0} / M_{P}\right)=1.22 \times 10^{-61}\left(h_{0} / 0.7\right)$. In terms of the typical values of the various

\footnotetext{
${ }^{19}$ In equation (5.2) we traded $\epsilon$ for the tensor scalar ratio $r_{\mathrm{T}}$ by using the consistency relation $r_{\mathrm{T}}=16 \epsilon$.
} 

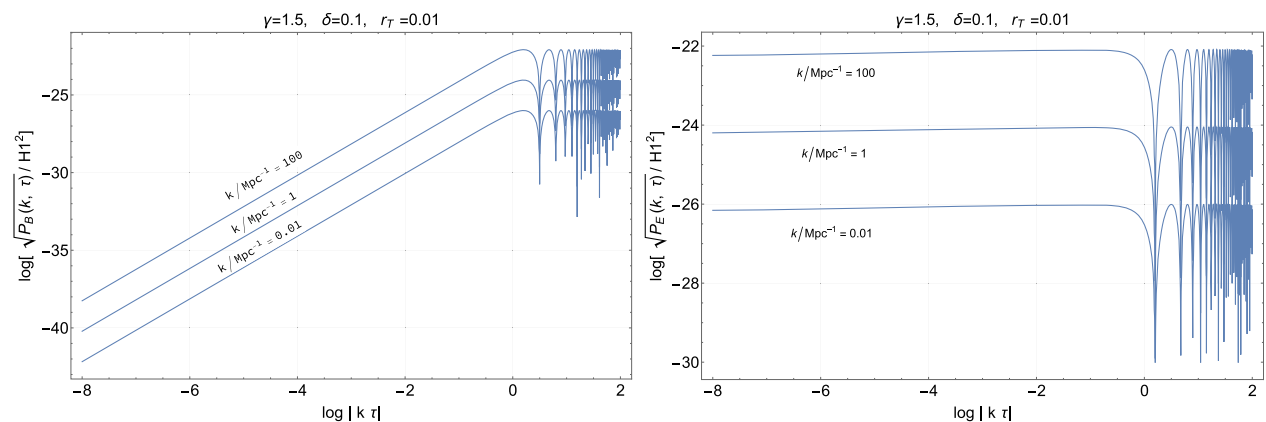

Figure 3. We illustrate the comoving power spectra prior to reentry for a particular choice of the parameters and for three different comoving scales.

parameters, equation (5.3) can also be written in the following revealing form:

$$
\frac{k}{a_{1} H_{1}}=10^{-23.05}\left(\frac{k}{\mathrm{Mpc}^{-1}}\right)\left(\frac{r_{\mathrm{T}}}{0.01}\right)^{-1 / 4}\left(\frac{h_{0}^{2} \Omega_{R 0}}{4.15 \times 10^{-5}}\right)^{-1 / 4}\left(\frac{\mathcal{A}_{\mathcal{R}}}{2.41 \times 10^{-9}}\right)^{-1 / 4} \zeta_{r}^{1 / 2-\alpha} .
$$

In equations (5.3) and (5.4) $\zeta_{r}=H_{r} / H_{1}$ and $H_{r}$ is the value of the Hubble rate at the moment when the radiation starts dominating; in the concordance paradigm $\zeta_{r}=1$ (and this is the value we shall assume most of the time). However it is sometimes useful to check for the stability of the obtained results with respect to a change of the reference scenario. This is why equations (5.3) and (5.4) have been derived by considering a generalized post-inflationary evolution where the radiation epoch is preceded by a phase expanding at a rate parametrized ${ }^{20}$ by $\alpha$. Finally, throughout the explicit numerical evaluation it will be useful to bear in mind the following two general expressions:

$$
\left(\frac{a_{1}}{a_{0}}\right)=\zeta_{r}^{\alpha-1 / 2}\left(\frac{2 \Omega_{R 0}}{\pi \epsilon \mathcal{A}_{\mathcal{R}}}\right)^{1 / 4} \sqrt{\frac{H_{0}}{M_{P}}}, \quad\left(\frac{a_{0} H_{0}}{a_{1} H_{1}}\right)=\zeta_{r}^{1 / 2-\alpha}\left(2 \pi \epsilon \Omega_{R 0} \mathcal{A}_{\mathcal{R}}\right)^{-1 / 4} \sqrt{\frac{H_{0}}{M_{P}}} .
$$

\subsection{Physical spectra prior to reentry}

In the previous sections we discussed mainly the comoving spectra of the hyperelectric and hypermagnetic fields but in various situations the distinction between the physical and the comoving fields has played a crucial role. The distinction between physical and comoving power spectra has been given in equation (2.27) and here we just want to illustrate, in a preliminary respective, the evolution of the comoving fields as a function of $k \tau$. In figure 3 we illustrate the evolution of the common logarithm of the square roots of the hyperelectric and hyperamagnetic power spectra ${ }^{21}$ as a function of the common logarithm of $|k \tau|$. The values of $\gamma$ and $\delta$ have been randomly chosen since the parameter space will be more carefully scrutinised in subsection 5.5. In figure 3 three different scales have been included. The most relevant

\footnotetext{
${ }^{20}$ If this intermediate stage of expansion is dominated by a perfect fluid with barotropic index $w$, we have that $\alpha=2 /[3(w+1)]$.

${ }^{21}$ The electromagnetic projection of the hypercharge through the Weinberg angle has not been included but it will be carefully taken into account when charting the parameter space in subsection 5.5.
} 
one is the scale of the primordial gravitational collapse (i.e. $k=\mathcal{O}\left(\mathrm{Mpc}^{-1}\right)$ ). The other two scales are just illustrative but the same bunch of wavenumbers will be illustrated in the contour plots of subsection 5.5.

For $\tau \ll \tau_{k}$ the power spectra follow from equations (4.20), (4.21) and (4.43), (4.44) evaluated in the limit $k \tau \ll 1$. When the gauge coupling increases the physical spectra (2.27) associated with the comoving expressions of equations (4.20) and (4.21) $\operatorname{are}^{22}$ :

$$
\begin{aligned}
& P_{B}^{\text {(phys) }}(k, \tau)=\frac{H_{1}^{4}}{\lambda_{1}}\left(\frac{a_{1}}{a}\right)^{4} \frac{D(\gamma+1 / 2)}{(2 \delta+1)^{2} q^{4 \delta}}\left(\frac{k}{a_{1} H_{1}}\right)^{6-2 \gamma}\left(\frac{a_{1} H_{1}}{a H}\right)^{4 \delta+2}, \\
& P_{E}^{\text {(phys) }}(k, \tau)=\frac{H_{1}^{4}}{\lambda_{1}}\left(\frac{a_{1}}{a}\right)^{4} \frac{D(\gamma+1 / 2)}{q^{4 \delta}}\left(\frac{k}{a_{1} H_{1}}\right)^{4-2 \gamma}\left(\frac{a_{1} H_{1}}{a H}\right)^{4 \delta}, \quad \tau<\tau_{k} .
\end{aligned}
$$

When the gauge coupling decreases the comoving spectra of equations (4.43) and (4.44) must be inserted into equation (2.27) and the result is:

$$
\begin{aligned}
& \widetilde{P}_{B}^{\text {(phys) }}(k, \tau)=\frac{H_{1}^{4}}{\lambda_{1}}\left(\frac{a_{1}}{a}\right)^{4} \frac{D(\widetilde{\gamma}+1 / 2)}{q^{4 \widetilde{\delta}}}\left(\frac{k}{a_{1} H_{1}}\right)^{4-2 \widetilde{\gamma}}\left(\frac{a_{1} H_{1}}{a H}\right)^{4 \widetilde{\delta}}, \\
& \widetilde{P}_{E}^{\text {(phys) }}(k, \tau)=\frac{H_{1}^{4}}{\lambda_{1}}\left(\frac{a_{1}}{a}\right)^{4} \frac{D(\widetilde{\gamma}+1 / 2)}{(2 \widetilde{\delta}+1)^{2} q^{4 \widetilde{\delta}}}\left(\frac{k}{a_{1} H_{1}}\right)^{6-2 \widetilde{\gamma}}\left(\frac{a_{1} H_{1}}{a H}\right)^{4 \widetilde{\delta}+2}, \quad \tau<\tau_{k} .
\end{aligned}
$$

The explicit formulas of equations (5.2), (5.3) and (5.5) allow for an explicit evaluation of equations (5.6), (5.7) and (5.8), (5.9). If $\lambda_{1}=\mathcal{O}(1)$ the only spectra consistent with the perturbative evolution of the gauge coupling are the ones of equations (5.6) and (5.7). In the case of decreasing gauge coupling $\lambda_{1}$ must be instead extremely large (see also equation (2.44) and discussion therein). This means, however, that the magnetic spectrum (5.8) will be suppressed as $\lambda_{1}^{-1} \simeq e^{-2 \widetilde{\gamma} N}$ where $N$ now denotes the total number of inflationary $e$-folds. Let us finally remark that in the sudden limit (i.e. $\delta \rightarrow 0$ and $\widetilde{\delta} \rightarrow 0$ ) the physical power spectra will always follow from equation (2.27) but the related comoving spectra will be given, respectively, by equations (4.24), (4.25) and (4.45), (4.46). For instance, in the case of increasing gauge coupling the result will be:

$$
\begin{aligned}
& P_{B}^{\text {(phys) }}(k, \tau)=\frac{H_{1}^{4}}{\lambda_{1}}\left(\frac{a_{1}}{a}\right)^{4} D(\gamma+1 / 2)\left(\frac{k}{a_{1} H_{1}}\right)^{6-2 \gamma}\left(\frac{a_{1} H_{1}}{a H}\right)^{2}, \\
& P_{E}^{\text {(phys) }}(k, \tau)=\frac{H_{1}^{4}}{\lambda_{1}}\left(\frac{a_{1}}{a}\right)^{4} D(\gamma+1 / 2)\left(\frac{k}{a_{1} H_{1}}\right)^{4-2 \gamma}, \quad \tau<\tau_{k} .
\end{aligned}
$$

\subsection{Physical spectra after reentry}

For $\tau \geqslant \tau_{k}$ the evolution of the mode functions is modified by the presence of the conductivity and it is approximately given by ${ }^{23}$ :

$$
g_{k}^{\prime}=-k^{2} f_{k}-\sigma g_{k}, \quad f_{k}^{\prime}=g_{k} .
$$

\footnotetext{
${ }^{22}$ Concerning equations (5.6) and (5.7) we note that $\lambda_{1}=\lambda\left(-\tau_{1}\right)$. For the sake of simplicity we shall assume $\lambda_{1}=1$ even if $\lambda_{1}$ could also be slightly larger than 1 .

${ }^{23}$ Note that in a different system of units (where the gauge coupling is defined without the $\sqrt{4 \pi}$ factor) we would have $4 \pi \sigma$ (and not simply $\sigma$ ) in equation (5.12). We are assuming here that the gauge coupling does not evolve anymore and it is frozen to its constant value.
} 
These equations can be systematically solved as an expansion in $(k / \sigma)$ by setting initial conditions at $\tau=\tau_{k}$. To lowest order the solution of equation (5.12) for $\tau \geqslant \tau_{k}$ is:

$$
f_{k}(\tau)=A_{g f}\left(k, \tau_{1}, \tau_{k}\right) \frac{\bar{g}_{k}}{k} \mathrm{e}^{-\frac{k^{2}}{k_{\sigma}^{2}}}, \quad g_{k}(\tau)=\left(\frac{k}{\sigma}\right) A_{g g}\left(k, \tau_{1}, \tau_{k}\right) \bar{g}_{k} \mathrm{e}^{-\frac{k^{2}}{k_{\sigma}^{2}}}
$$

where the magnetic diffusivity scale $k_{\sigma}$ has been defined as $k_{\sigma}^{-2}=\int_{\tau_{k}}^{\tau} \mathrm{d} z / \sigma(z)$. While the estimate of $k_{\sigma}$ can be made accurate by computing the transport coefficients of the plasma in different regimes, for the present purposes this is not necessary since the ratio $\left(k / k_{\sigma}\right)^{2}$ is so small, for the phenomenologically interesting scales, that the negative exponentials in equation (5.13) evaluate to 1 . In fact by taking $\tau=\tau_{\text {eq }}$ we have that $k_{\sigma}$ can be estimated as:

$$
\left(\frac{k}{k_{\sigma}}\right)^{2}=\frac{4.75 \times 10^{-26}}{\sqrt{2 h_{0}^{2} \Omega_{M 0}\left(z_{\mathrm{eq}}+1\right)}}\left(\frac{k}{\mathrm{Mpc}^{-1}}\right)^{2},
$$

where $\Omega_{M 0}$ is the present critical fraction in matter and $z_{\text {eq }}+1=a_{0} / a_{\text {eq }} \simeq \mathcal{O}(3200)$ is the redshift of matter-radiation equality. The physical power spectra after for $\tau \gg \tau_{k}$ will then be given by:

$$
\begin{aligned}
& P_{B}^{\text {(phys) }}(k, \tau)=H_{1}^{4}\left(\frac{a_{1}}{a}\right)^{4} D(\gamma+1 / 2)\left(\frac{k}{a_{1} H_{1}}\right)^{4-2 \gamma-2 \delta} F_{B}\left(k \tau_{k}, \delta\right) \mathrm{e}^{-2 \frac{k^{2}}{k_{\sigma}^{2}}}, \\
& P_{E}^{\text {(phys) }}(k, \tau)=\left(\frac{k}{\sigma}\right)^{2} H_{1}^{4}\left(\frac{a_{1}}{a}\right)^{4} D(\gamma+1 / 2)\left(\frac{k}{a_{1} H_{1}}\right)^{4-2 \gamma-2 \delta} F_{E}\left(k \tau_{k}, \delta\right) \mathrm{e}^{-2 \frac{k^{2}}{k_{\sigma}^{2}}},
\end{aligned}
$$

where $F_{B}(x, \delta)$ and $F_{E}(x, \delta)$ coincide with the ones defined in equations (4.20) and (4.21).

According to equations (5.15) and (5.16) the magnetic spectrum for $k<k_{\sigma}$ is practically not affected by the conductivity while the electric power spectrum is suppressed by $k^{2} / \sigma^{2} \ll 1$. Since prior to decoupling the electron-photon and electron-proton interactions tie the temperatures close together, the conductivity scales as $\sigma \sim \sqrt{T / m_{\mathrm{e}}} T / \alpha_{\mathrm{em}}$ where $m_{\mathrm{e}}$ is the electromagnetic mass, $T$ is the temperature and $\alpha_{\mathrm{em}}$ is the fine structure constant. For instance, if we take $T=\mathcal{O}(\mathrm{eV})$ we get $k / \sigma=\mathcal{O}\left(10^{-30}\right)$ for $k=\mathcal{O}\left(\mathrm{Mpc}^{-1}\right)$. The electric power spectrum of equation (5.16) will then be suppressed, in comparison with the magnetic spectrum, by a factor ranging between 40 and 60 orders of magnitude. This also demonstrates, in practical terms, that the duality symmetry is broken for $\tau>\tau_{k}$.

\subsection{Magnetogenesis constraints}

According to the standard lore the observed large-scale fields in galaxies (and to some extent in clusters) should have been much smaller before the gravitational collapse of the protogalaxy. Compressional amplification typically increases the initial values of the magnetic seeds by 4 or even 5 orders of magnitude and the logic underlying this statement is in short the following (see e.g. [19-21]). When the protogalactic matter collapsed by gravitational instability over a typical scale $\mathcal{O}(\mathrm{Mpc})$ the mean matter density before collapse was of the order of $\rho_{\text {crit }}$ whereas right after the collapse the mean matter density became, approximately, six orders of magnitude larger than the critical density. Since the physical size of the patch decreases from $1 \mathrm{Mpc}$ to $30 \mathrm{kpc}$ the magnetic field increases, because of flux conservation, of a factor $\left(\rho_{a} / \rho_{b}\right)^{2 / 3} \sim 10^{4}$ where $\rho_{a}$ and $\rho_{b}$ are, respectively the energy densities right after and right before gravitational collapse. Most of the work in the context of the dynamo theory focuses on reproducing the correct features of the magnetic field of our Galaxy. The dynamo term may be responsible for the origin of the magnetic field of the Galaxy. The Galaxy has a typical rotation period of 
Table 1. Numerical values of the magnetic power spectrum at different scales and in the framework of the $(\gamma, \delta)$ transition where the gauge coupling is perturbative throughout all the stages of its evolution.

\begin{tabular}{llll}
\hline & $\gamma=1.8, \delta=0.01$ & $\gamma=1.9, \delta=0.01$ & $\gamma=2, \delta=0.01$ \\
\hline$k=1 \mathrm{Mpc}^{-1}$ & $\sqrt{P_{B}^{(\text {phys })}}=10^{-7.3} \mathrm{nG}$ & $\sqrt{P_{B}^{(\text {phys })}}=10^{-4.9} \mathrm{nG}$ & $\sqrt{P_{B}^{(\text {phys }}}=10^{-2.30} \mathrm{nG}$ \\
$k=0.1 \mathrm{Mpc}^{-1}$ & $\sqrt{P_{B}^{(\text {phys })}}=10^{-7.25} \mathrm{nG}$ & $\sqrt{P_{B}^{(\text {phys }}}=10^{-4.78} \mathrm{nG}$ & $\sqrt{P_{B}^{(\text {phys }}}=10^{-2.29} \mathrm{nG}$ \\
$k=0.01 \mathrm{Mpc}^{-1}$ & $\sqrt{P_{B}^{(\text {phys })}}=10^{-7.44} \mathrm{nG}$ & $\sqrt{P_{B}^{(\text {phys })}}=10^{-4.87} \mathrm{nG}$ & $\sqrt{P_{B}^{(\text {phys })}}=10^{-2.28} \mathrm{nG}$ \\
\hline
\end{tabular}
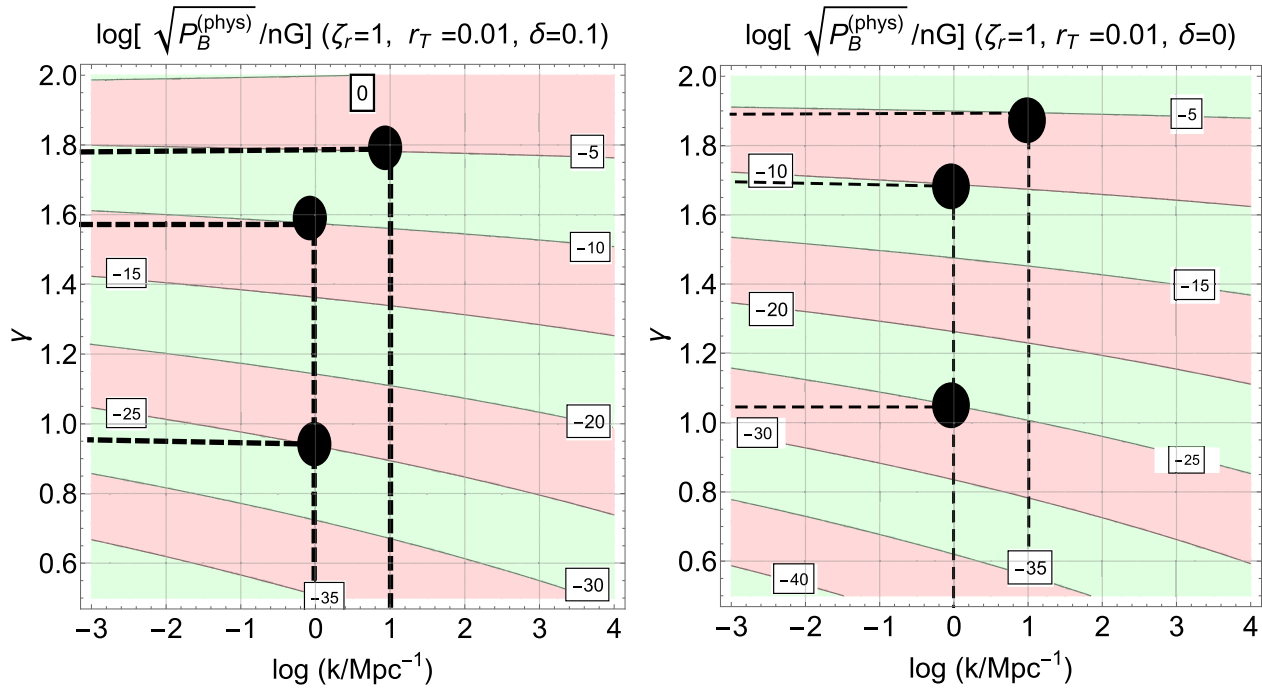

Figure 4. The parameter space is illustrated in the $(\gamma, k)$ plane. The numbers appearing on the various contours correspond to the common logarithm of the physical power spectrum. The common logarithm of $k$ in units of $\mathrm{Mpc}^{-1}$ is reported on the horizontal axis.

$3 \times 10^{8}$ years and comparing this figure with the typical age of the Galaxy, $\mathcal{O}\left(10^{10}\right.$ years $)$, it can be appreciated that the Galaxy performed about 30 rotations since the time of the protogalactic collapse. The achievable amplification produced by the dynamo instability can be at most of $10^{13}$, i.e. $e^{30}$. Thus, if the present value of the galactic magnetic field is $\mathcal{O}(\mu \mathrm{G})$, its value right after the gravitational collapse of the protogalaxy might have been as small as $\mathcal{O}\left(10^{-10}\right) \mathrm{nG}$ over a typical scale of $30-100 \mathrm{kpc}$.

The compressional amplification and the dynamical action are typically combined together so that the magnetogenesis requirements roughly demand that the magnetic fields at the time of the gravitational collapse of the protogalaxy should be approximately larger than a (minimal) power spectrum which can be estimated between $\mathcal{O}\left(10^{-32}\right) \mathrm{nG}^{2}$ and $\mathcal{O}\left(10^{-22}\right) \mathrm{nG}^{2}$ :

$$
\log \left(\frac{\sqrt{P_{B}^{\text {(phys) }}}}{\mathrm{nG}}\right) \geqslant-\xi, \quad 11<\xi<16
$$


The least demanding requirement of equation (5.17) (i.e. $\sqrt{P_{B}^{(\text {phys }}} \geqslant 10^{-16} \mathrm{nG}$ ) follows by assuming that, after compressional amplification, every rotation of the Galaxy increases the initial magnetic field of one $e$-fold. According to some this requirement is not completely since it takes more than one $e$-fold to increase the value of the magnetic field by one order of magnitude and this is the rationale for the most demanding condition of equation (5.17), i.e. $\sqrt{P_{B}^{\text {(phys) }}} \geqslant 10^{-11} \mathrm{nG}$.

\subsection{Charting the parameter space}

Let us now consider a simplified estimate where $\zeta_{r} \rightarrow 1$ and $\alpha \rightarrow 0$. This is the situation of the concordance paradigm and for the typical values of the parameters given above the results for $\sqrt{P_{B}^{\text {(phys) }}\left(k, \tau_{0}\right)}$ are reported in table 1 for different values of $\gamma$ and $k$. Since the non-screened vector modes of the hypercharge field project on the electromagnetic fields through the cosine of the Weinberg angle the estimates of table 1 follow from equation (5.15) after multiplying the obtained result by $\cos \theta_{\mathrm{W}}$ (recall, in this respect, that $\sin ^{2} \theta_{\mathrm{W}} \simeq 0.22$ ). The illustrative orders of magnitude of table 1 will now be complemented with more detailed estimates allowing for a simultaneous variation of the different parameters in the light of the typical figures required by the magnetogenesis considerations.

We shall distinguish the results obtained in the smooth (i.e. $\delta \ll \gamma$ ) and the sudden (i.e. $\delta \rightarrow 0$ ) approximations. In practice this will be achieved by illustrating all the subsequent numerical results for $\delta=0.1$ and the case $\delta=0$. In figure 4 the physical power spectrum is illustrated in the $(k, \gamma)$ plane. The black blobs in both plots indicate three reasonable regions of the parameter space where all the pertinent phenomenological constraints are satisfied. The numbers on the various curves denote, as explained on top of the plot, the common logarithms of $\sqrt{P_{B}^{(\text {phys })}}$ (expressed in $\mathrm{nG}$ ) along that curve. By looking at the intercept on the $\gamma$ axis we see that the phenomenologically reasonable values of $\gamma$ correspond to spectra that are blue or, at most, quasi-flat but always slightly increasing with $k$ (as already suggested by the results of table 1).

The results of figure 4 are complemented by figure 5 where the power spectra are illustrated in the $\left(\gamma, r_{\mathrm{T}}\right)$ plane for fixed wavenumber $k=\mathrm{Mpc}^{-1}$. The black blobs correspond this time to the regions where $r_{\mathrm{T}}=\mathcal{O}(0.01)$ : for instance in reference [50] the combination of different data sets implies $r_{\mathrm{T}}<0.07$ while in reference [51] the range of values $r_{\mathrm{T}}<0.064$ is quoted. By looking simultaneously at figures 4 and 5 we can appreciate that the viable range of $\gamma$ roughly extends between 1.3 and 2 .

\subsection{Post-inflationary phases preceding the radiation epoch and reheating dynamics}

The results obtained so far also follow the continuity of the extrinsic curvature across the inflationary boundary. This approximation is customarily dubbed instant reheating and it is often used when setting bounds on quantities like the number of inflationary $e$-folds and the tensor to scalar ratio (see e.g. $[50,51]$ ). The explosive particle decay as well as other non-perturbative effects related to the preheating dynamics probably bring the situation closer to the case of an instant reheating (see e.g. [52, 53]). However, if the reheating is perturbative as originally suggested in the new inflationary scenario [54] the effect of the duration of the reheating phase may be relevant (see also [47]).

In figures 6-8 we illustrated the situation where the dominance of radiation is preceded by a post-inflationary phase extending down to the relatively small values of the curvature scale. We remind from equations (5.3) and (5.4) that $\zeta_{r}=H_{r} / H_{1}$ and $H_{r}$ denotes the Hubble rate at 

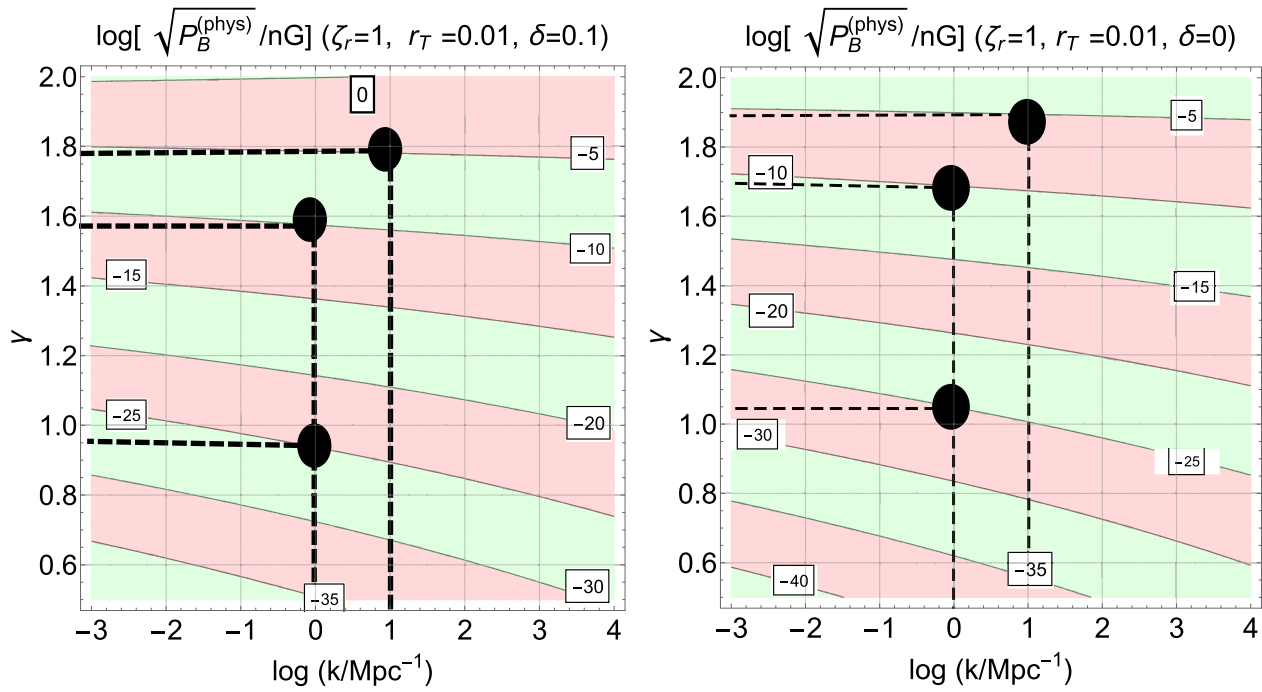

Figure 5. We illustrate the common logarithm of the power spectrum in the plane $\left(\gamma, r_{\mathrm{T}}\right)$. We remind that $r_{\mathrm{T}}$ denotes, within the present notations, the tensor to scalar ratio.
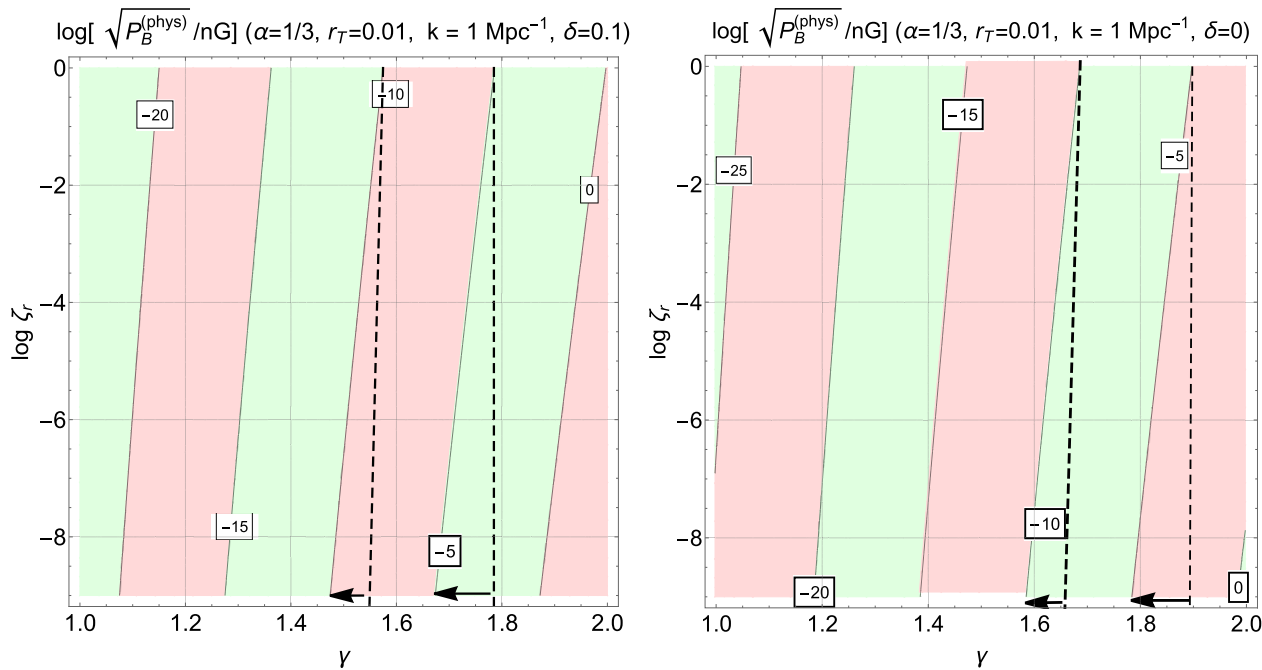

Figure 6. We illustrate the parameter space in the $\left(\gamma, \zeta_{r}\right)$ plane for a typical expansion rate $\alpha=1 / 3$ corresponding to a post-inflationary stiff phase.

the onset of the radiation dominance. Since $\alpha$ measures the expansion rate between $H_{1}$ and $H_{r}$ the parameter space of the model can also be investigated by varying $\alpha$ and $\zeta_{r}$. For instance in figure 6 the value of $\alpha$ has been fixed (i.e. $\alpha \rightarrow 1 / 3$ as indicated in each of the two plots) and the contours of constant magnetic spectrum ${ }^{24}$ have been investigated in the $\left(\gamma, \zeta_{r}\right)$ plane. It is

${ }^{24}$ We remind that the values of the magnetic power spectrum reported on each of the curves remain the same all along the curve since this is the meaning of a contour plot. 

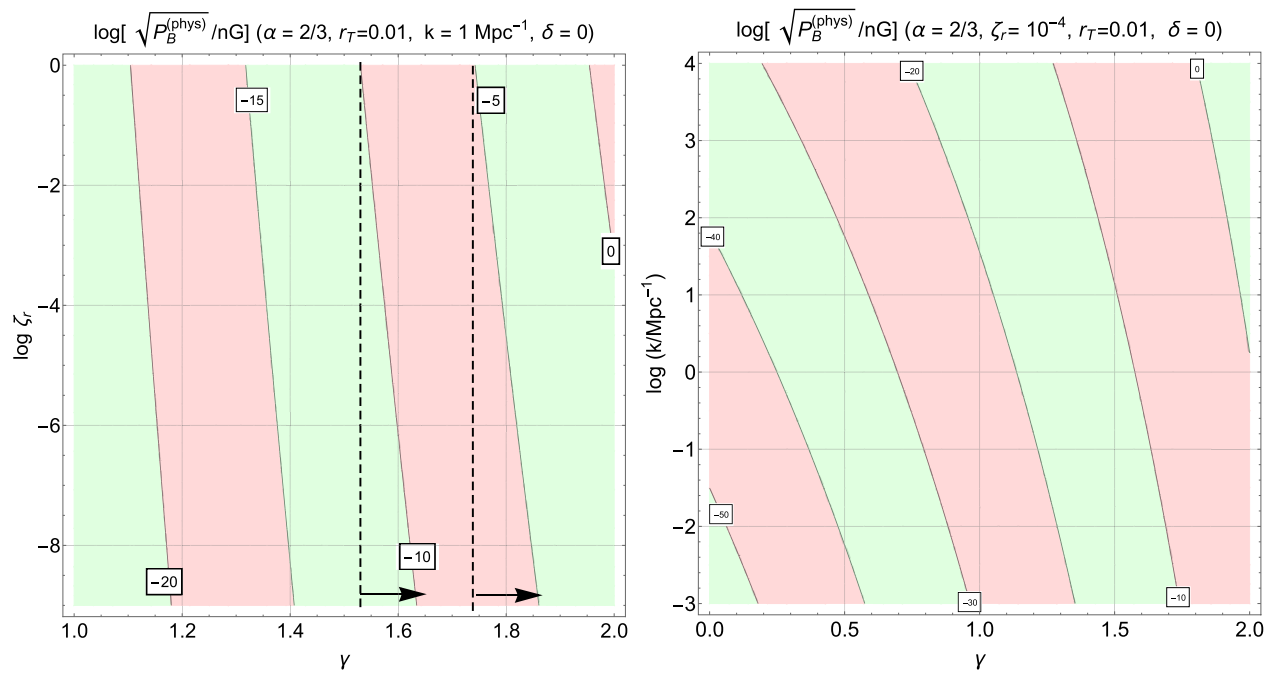

Figure 7. In the plot at the left the parameter space is illustrated in the $\left(\gamma, \zeta_{r}\right)$ plane while in the plot at the right the parameter space is instead illustrated in the $(\gamma, k)$ plane. In both plots the typical expansion rate is faster than radiation (i.e. $\alpha=2 / 3$ ) as it happens, for instance, in the context of the reheating dynamics.
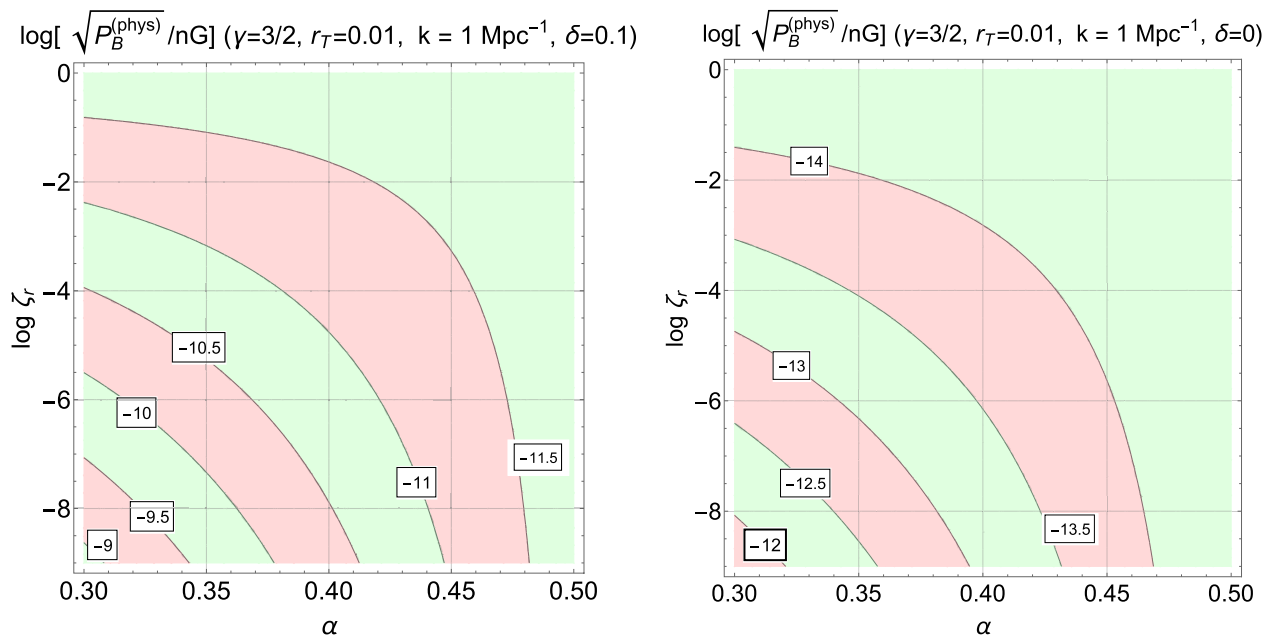

Figure 8. The allowed region of the parameter space is illustrated $\left(\alpha, \zeta_{r}\right)$ plane for a fixed value of $\gamma$.

now relevant to consider with care the dashed lines in figure 6: we see that the same amplitude of the magnetic power spectrum associated to a certain value of $\gamma$ when $\zeta_{r} \rightarrow 1$ (i.e. in the absence of an intermediate phase) can be obtained for a comparatively lower value of $\gamma$ if the intermediate phase is present (i.e. $\zeta_{r}<1$ ). This is the meaning of the arrows appearing in figure 6: for $\alpha=1 / 3$ (and for a given $\zeta_{r}<1$ ) the $\gamma$ required to obtain a certain value of the power spectrum is comparatively smaller than in the case $\zeta_{r}=1$. This conclusion depends on 
the value of $\alpha$ : in figure 6 the case $\alpha=1 / 3$ is meant to represent a post-inflationary phase dominated by a stiff fluid expanding at a rate slower than radiation.

In the case of an extended reheating dynamics the expansion rate prior to the dominance of radiation is expected to be faster than in the stiff case. In figure 7 we then considered the possibility of a post-inflationary phase preceding the ordinary radiation phase where the expansion rate is effectively faster than radiation. By looking at the dashed lines in figure 7 we see that the situation differs from the one illustrated in figure 6: for $\alpha=2 / 3$ (and for a given $\zeta_{r}<1$ ) the $\gamma$ required to obtain a certain value of the power spectrum is comparatively larger than in the case $\zeta_{r}=1$. The same conclusion holds for different expansion rates faster than radiation. Along this perspective we can therefore consider the plane $\left(\alpha, \zeta_{r}\right)$ for a fixed value of $\gamma$ (e.g. $\gamma \rightarrow 3 / 2$, as in figure 8). As $\zeta_{r}$ diminishes (i.e. the post-inflationary phase gets longer) the power spectrum gets larger. In figure 8 we considered the situation where the expansion rate during the intermediate phase was slower than radiation and we have also purposely chosen $\gamma=3 / 2$. For larger values of $\gamma$ the amplitude of the power spectra will be larger but the basic trend of figure 8 will remain the same. All in all we can therefore say that the inclusion of a post-inflationary phase preceding the ordinary radiation-dominated stage has a threefold effect depending on the expansion rate:

- If the post-inflationary phase preceding radiation expands faster than radiation the amplitude of the power spectrum gets mildly reduced for a fixed value of $\gamma$; this means that to achieve the same amplitude we should have a comparatively larger value of $\gamma$;

- If the post-inflationary phase expands at a rate slower than radiation (as it happens in the case of stiff post-inflationary phases) the power spectrum is mildly enhanced, i.e. to achieve the same spectral amplitude we should have a comparatively smaller value of $\gamma$;

- In both cases the length of the post-inflationary phase is crucial: for a long stiff postinflationary phase the amplitude of the magnetic power spectrum gets larger; conversely for a delayed reheating expanding faster than radiation the power spectrum gets reduced.

We finally mention that the effects of a post-inflationary phase have been qualified as mild in the previous list of items. By this we simply mean that for an excursion of $\zeta_{r}$ of about 9 orders of magnitude the spectral index gets renormalised by a factor of the order of 0.1 . So this preliminary analysis seems to confirm that these are not large effects.

\subsection{Final assessment of the obtained results and the meaning of the parameter space}

Since this analysis has not been done before it is appropriate, as we conclude, to summarize the main results. The first aspect we stress is that has been be explicitly used to deduce the gauge power spectra both during inflation and at late times. The symmetry can therefore be used to verify and constrain the explicit expressions of the power spectra.

If the gauge coupling increases during a quasi-de Sitter stage of expansion only the hyperelectric spectrum can be flat for typical wavelengths larger than the effective horizon during inflation (see e.g. equation (3.13) and discussions therein). The duality symmetry implies therefore that only the hypermagnetic spectrum can be flat (see equation (3.28)) during a quasi-de Sitter stage of expansion and for typical wavelengths larger than the effective horizon. These results, even if not explicitly related to the duality symmetry, were known also in references [23] and suggested the idea that to have a successful magnetogenesis scenario we should necessarily consider a dynamical situation where, in the language of this paper, the gauge coupling decreases. Only in this case we could obtain a quasi-flat magnetic spectrum during the quasi-de Sitter phase. Even if it has been later on recognised that non only the inflaton but also some other spectator fields could effectively play the role of a dynamical gauge coupling [24] the 
conclusion remained: only if the gauge coupling decreases a flat hypermagnetic spectrum can be obtained during inflation.

The first observation is therefore that, within the present approach the twofold conclusion mentioned in the previous paragraph, follows directly from the dual treatment of the problem and of the related spectra; see, in particular the discussions after equations (3.11), (3.12) and also after equations (3.26), (3.27). For a number of years, it has been assumed that only when the gauge coupling decreases the magnetogenesis constraints could be satisfied [25]. This possibility has however some drawbacks that have been accurately discussed here (see subsections 2.4.1 and 2.4.2): if the hypermagnetic spectrum is flat during inflation strong coupling is expected when the gauge coupling starts its evolution.

The main idea of this paper has been to consider a more complete scenario where the gauge coupling first increases (or decreases) and then flattens out at late times as illustrated in figures 1 and 2. This approach, heavily based on the continuity of the mode functions and of the extrinsic curvature also considers specifically the transient regime where the gauge coupling relaxes and it does it in a computable manner. In the past the common approximation was that the gauge coupling (and hence $\sqrt{\lambda}$ ) abruptly freezes after inflation: we argued that this approach is not fully consistent a priori since it implies that the relevant pump fields (like $\sqrt{\lambda^{\prime \prime}}$ ) are singular across the inflationary boundary. If the gauge coupling flattens out in a smooth manner the present results showed that the late-time hypermagnetic power spectra outside the horizon in the radiation epoch are determined by the hyperelectric fields at the end of inflation whereas the opposite is true in the case of decreasing coupling. The obtained results then suggest that a slightly blue hyperelectric spectrum during inflation may lead to a quasi-flat hypermagnetic spectrum prior to matter radiation equality and before the relevant wavelengths reenter the effective horizon. Once more these results are a consequence of duality and of a very accurate analysis of the transition regime between the early inflationary stage and the subsequent evolution, as illustrated in figures 1 and 2 . The obtained magnetic fields have been confronted with the magnetogenesis requirements and we can then conclude that large-scale magnetic fields can be generated during a quasi-de Sitter stage of expansion while the gauge coupling remains perturbative throughout all the stages of the dynamical evolution.

The results of this analysis have been summarized in figures 9 and 10. In figure 9 the shaded areas denote the region where the spectral energy density is subcritical both during and after inflation while the magnetogenesis and the cosmic microwave background (CMB) constraints are all satisfied. While the discussion of the $\mathrm{CMB}$ constraints would probably require a separate analysis, some general results could be used and these are essentially the ones reproduced in figure 6: they amount to requiring that the physical power spectrum after equality but before decoupling is smaller than $10^{-2} \mathrm{nG}$ for typical wavenumbers comparable with the pivot scale $k_{p}=0.002 \mathrm{Mpc}^{-1}$ at which the scalar and tensor power spectra are customarily assigned. The proper analysis of the $\mathrm{CMB}$ effects associated with the magnetic random fields started already after the first releases of the WMAP data; through the years various direct constraints have been derived both from the temperature and from the polarization anisotropies (see, for instance, [55] for a recent review). The main difference between the two plots of figure 9 stems from the magnetogenesis requirements: the larger area in the right plot corresponds to the situation where each galactic rotation amplifies the initial magnetic field value by one $e$-fold while the smaller area follows by requiring that the physical power spectrum exceed $10^{-22} \mathrm{nG}^{2}$ (see equation (5.17) and discussion therein). In figure 10 we consider the same constraints of figure 9 but in the $(\gamma, \delta)$ plane. The physical region obviously corresponds to the case $\delta \ll 1$.

All in all we can conclude that magnetogenesis is phenomenologically viable in the perturbative regime provided the gauge coupling increases during a quasi-de Sitter stage and then flattens out after the end of the inflationary phase. The sweet spot of the parameter space (i.e. 

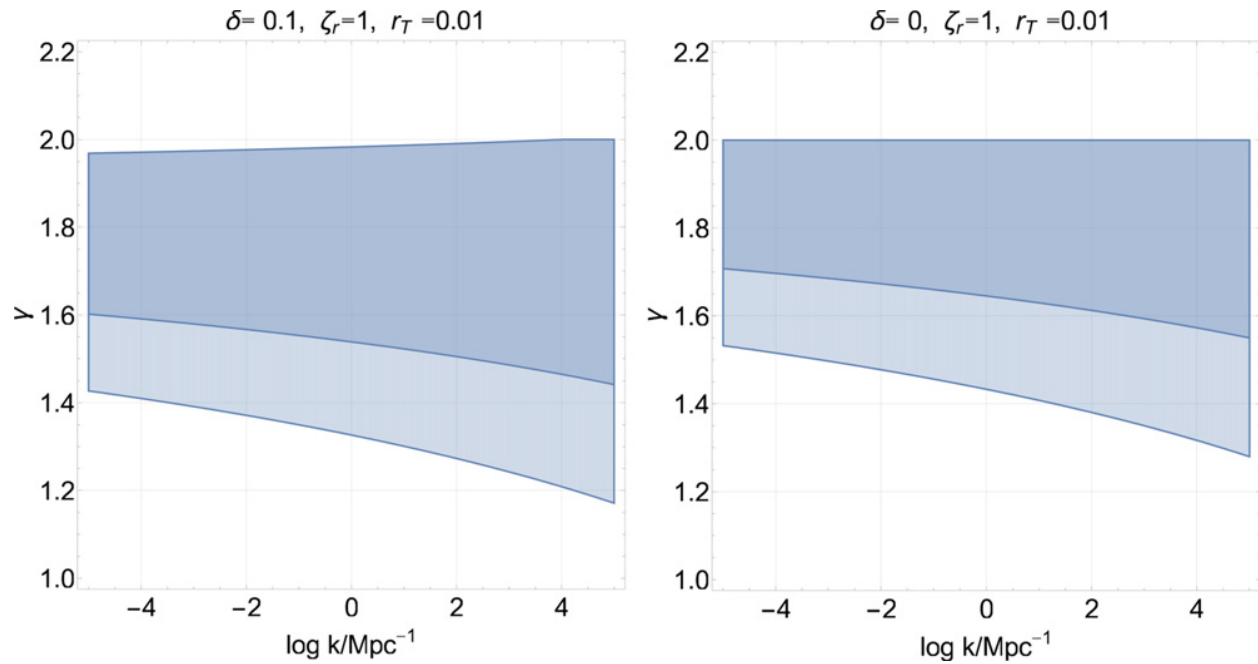

Figure 9. The allowed region of the parameter space is illustrated with a shaded area for fixed $\delta$ and in the $(\gamma, k)$ plane. The different shading corresponds to the two extreme values of $\xi$ appearing in equation (5.17).
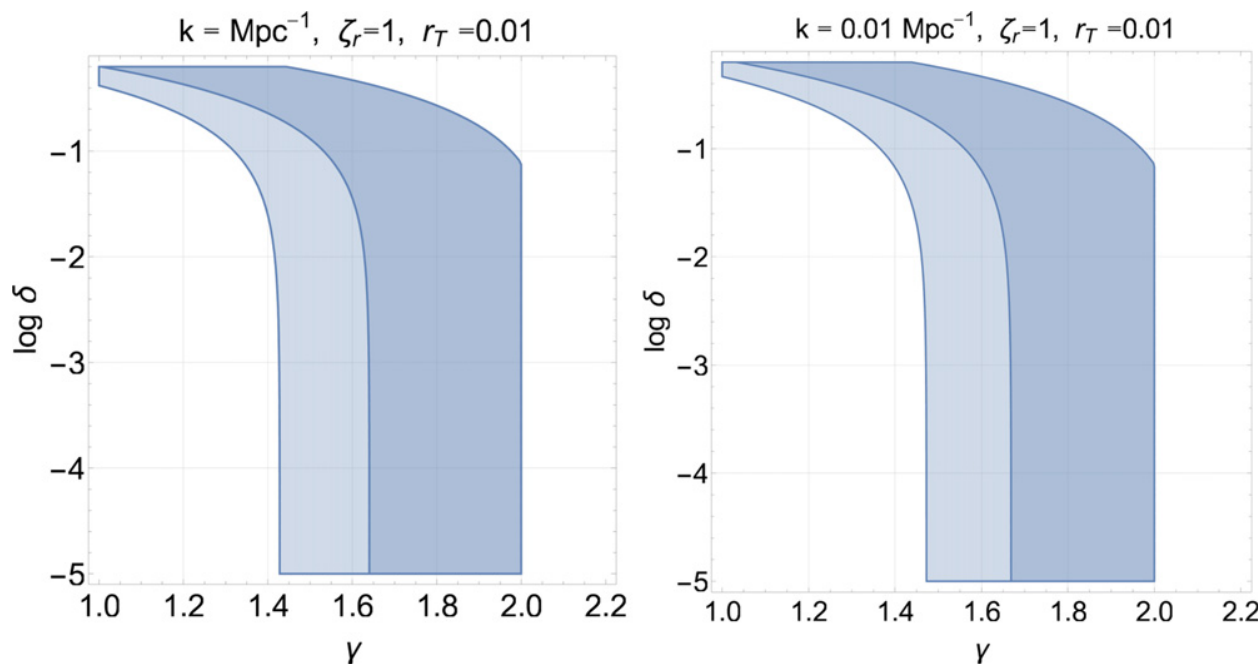

Figure 10. The allowed region of the parameter space is illustrated with a shaded area for fixed $k$ and in the $(\gamma, \delta)$ plane. As in figure 9 the different shadings correspond to the two extreme values of $\xi$ appearing in equation (5.17).

where all reasonable constraints are met) corresponds to a slightly increasing (blue) spectrum where $\mathcal{O}(1.6) \leqslant \gamma<2$. If the magnetogenesis requirement are relaxed $\gamma$ can be as small as $\mathcal{O}(1.4)$.

It does not make much sense to compare the present findings with the parameter space of the models where the gauge coupling decreases since, in those cases, a strongly coupled regime 
is expected at the onset of inflation. We instead conclude with a discussion of the various parameters of the scenario. These parameters are:

- $\gamma$ and $\delta$ (or $\widetilde{\gamma}$ and $\widetilde{\delta}$ in the dual situation) describing the dynamics of the gauge coupling according to figures 1 and 2 ;

- The maximal inflationary scale which is however determined by the tensor to scalar ratio $r_{\mathrm{T}}$ which we took smaller than about $0.64[50,51]$;

- The possible presence of a post-inflationary phase whose length has been parametrized by $\zeta_{r}$;

- The expansion rate during the latter phase.

To these parameters we could eventually add $e_{1}=\sqrt{4 \pi / \lambda_{1}}$, i.e. the value of the gauge coupling at the end of inflation but this is implicitly included in the initial profile of the gauge couplings of figures 4 and 5. Depending on how we count we therefore have overall 5 or 6 parameters. If we would consider the case of instantaneous reheating the number of parameters could be reduced by 2 . We are not interested here in the minimisation of the free parameters since the purpose of this paper is just to illustrate a potentially novel approach to the problem: more parameters might be included to account for more detailed physical aspects of the scenario. It is actually debatable if the minimisation of the parameters should be the ultimate purpose of a scenario and the sole criterion to assess its viability: an increase in the parameters typically entails a more accurate description of certain phenomena and a good example is actually the effect of reheating on magnetogenesis, as discussed above. Other examples could be made but it is not our purpose here to debate the heuristic value of this type of arguments.

\section{Concluding considerations}

Duality implies that the hypermagnetic power spectra parametrically amplified from quantum fluctuations during a quasi-de Sitter stage of expansion are scale-invariant (or slightly blue) if the gauge coupling decreases while an increase of the gauge coupling is only compatible with a flat hyperelectric spectrum for wavelengths larger than the effective horizon at the corresponding epoch. The same duality symmetry demands that the late-time gauge spectra do not always coincide with the results obtained at the end of inflation: the late-time hypermagnetic spectra follow directly from the hypermagnetic mode functions at the end of inflation whenever the gauge coupling decreases. Conversely if the gauge coupling increases the late-time hypermagnetic spectra are determined by the hyperelectric mode functions at the end of the inflationary phase. On a technical ground these results follow from the specific analysis of an appropriate transition matrix whose elements have well defined transformation properties under the duality symmetry and control the form of the late-time spectra.

Form a semiclassical viewpoint the mechanism analyzed here is the gauge analog of the Sakharov oscillations where traveling waves are transformed into standing waves with well defined phases that depend on the dynamics of the underlying geometry. The standing oscillations associated with the hyperelectric and with the hypermagnetic mode functions follow from the dynamics of the gauge couplings. Only when the gauge coupling decreases it is therefore reasonable to compute the post-inflationary physical spectra by a simple redshift of the comoving result during inflation. Conversely such a procedure would lead to an incorrect result in the case of an increasing gauge coupling where the late time hypermagnetic spectrum is determined by the hyperelectric spectrum at the end of inflation.

In summary, all the magnetogenesis constraints can be successfully satisfied when the gauge coupling remains perturbative throughout all the stages of its evolution. More precisely a slightly blue hyperelectric spectrum during inflation may lead to a quasi-flat hypermagnetic 
spectrum at late times. The induced large-scale magnetic fields turn out to be of the order of a few thousands of a $\mathrm{nG}$ over typical length scales between few Mpc and $100 \mathrm{Mpc}$. The magnetogenesis requirements are therefore satisfied together with all the backreaction constraints both during and after inflation. For the sweet spots of the parameter space the only further amplification required to seed the galactic magnetic fields is the one associated with the compressional amplification.

\section{Acknowledgments}

It is a pleasure to thank T Basaglia and S Rohr of the CERN Scientific Information Service for their kind help throughout various stages of this investigation.

\section{Data availability statement}

No new data were created or analysed in this study.

\section{Appendix A. Transition matrix and increasing gauge coupling}

In this first part of this appendix we shall consider a gauge coupling that sharply increases during the inflationary phase and then flattens out in the post-inflationary era. The profile of $\sqrt{\lambda}$ corresponds, in this case, to equations (2.33) and (2.34) with $0 \leqslant \delta \ll \gamma$ (see also figure 1 and discussion therein). Since the parametrization of $\sqrt{\lambda}$ is continuous and differentiable the evolution of the mode functions during the post-inflationary stage (i.e. for $\tau \geqslant-\tau_{1}$ ) follows from the solution of equations (2.20) and (2.21). For $\tau \geqslant-\tau_{1}$ the mode functions $f_{k}(\tau)$ and $g_{k}(\tau)$ are given in terms of certain coefficients $A_{ \pm}\left(k, \tau_{1}\right)$ :

$$
\begin{aligned}
f_{k}(\tau) & =\frac{\sqrt{k y}}{\sqrt{2 k}}\left[A_{-}\left(k, \tau_{1}\right) N_{\nu} H_{\nu}^{(1)}(k y)+A_{+}\left(k, \tau_{1}\right) N_{\nu}^{*} H_{\nu}^{(2)}(k y)\right], \\
g_{k}(\tau) & =\sqrt{\frac{k}{2}} \sqrt{k y}\left[A_{-}\left(k, \tau_{1}\right) N_{\nu} H_{\nu-1}^{(1)}(k y)+A_{+}\left(k, \tau_{1}\right) N_{\nu}^{*} H_{\nu-1}^{(2)}(k y)\right],
\end{aligned}
$$

where the following auxiliary quantities have been introduced:

$$
\begin{aligned}
N_{\nu} & =\sqrt{\frac{\pi}{2}} \mathrm{e}^{\mathrm{i} \pi(2 \nu+1) / 4}, \quad y(\tau)=\tau+\tau_{1}(1+q), \\
q & =q(\delta, \gamma)=\frac{\delta}{\gamma}, \quad \nu=\left(\delta+\frac{1}{2}\right) .
\end{aligned}
$$

The separate continuity of equations (3.1), (3.2) and (A.1), (A.2) across $\tau=-\tau_{1}$, determines the explicit forms of $A_{ \pm}\left(k, \tau_{1}\right)$ in the $\operatorname{case}^{25} \gamma>1 / 2$ :

$$
\begin{aligned}
& A_{-}\left(k, \tau_{1}\right)=\frac{\mathrm{i} \pi N_{\mu}}{4 N_{\nu}} \sqrt{q} x_{1}\left[H_{\mu}^{(1)}\left(x_{1}\right) H_{\nu-1}^{(2)}\left(q x_{1}\right)-H_{\mu+1}^{(1)}\left(x_{1}\right) H_{\nu}^{(2)}\left(q x_{1}\right)\right], \\
& A_{+}\left(k, \tau_{1}\right)=\frac{\mathrm{i} \pi N_{\mu}}{4 N_{\nu}^{*}} \sqrt{q} x_{1}\left[H_{\mu+1}^{(1)}\left(x_{1}\right) H_{\nu}^{(1)}\left(q x_{1}\right)-H_{\mu}^{(1)}\left(x_{1}\right) H_{\nu-1}^{(1)}\left(q x_{1}\right)\right], \quad \gamma>1 / 2,
\end{aligned}
$$

\footnotetext{
${ }^{25}$ The inflationary mode functions depend on the range of $\gamma$ (see, in in this respect, equations (3.1)-(3.3)). Therefore that equation (A.4) only holds for $\gamma>1 / 2$ while equation (A.5) holds for $0<\gamma<1 / 2$.
} 
where $x_{1}=k \tau_{1}$. For $0<\gamma<1 / 2$ the expressions of $A_{ \pm}\left(k, \tau_{1}\right)$ are instead obtained by matching equations (3.1) and (3.3) with equations (A.1) and (A.2):

$$
\begin{aligned}
& A_{-}\left(k, \tau_{1}\right)=\frac{\mathrm{i} \pi N_{\mu}}{4 N_{\nu}} \sqrt{q} x_{1}\left[H_{\mu}^{(1)}\left(x_{1}\right) H_{\nu-1}^{(2)}\left(q x_{1}\right)+H_{\mu-1}^{(1)}\left(x_{1}\right) H_{\nu}^{(2)}\left(q x_{1}\right)\right], \\
& A_{+}\left(k, \tau_{1}\right)=-\frac{\mathrm{i} \pi N_{\mu}}{4 N_{\nu}^{*}} \sqrt{q} x_{1}\left[H_{\mu-1}^{(1)}\left(x_{1}\right) H_{\nu}^{(1)}\left(q x_{1}\right)+H_{\mu}^{(1)}\left(x_{1}\right) H_{\nu-1}^{(1)}\left(q x_{1}\right)\right], \quad 0<\gamma<1 / 2 .
\end{aligned}
$$

Since the electric and the magnetic mode functions must obey the Wronskian normalization of equation (2.21), $A_{ \pm}\left(k, \tau_{1}\right)$ must satisfy the condition $\left|A_{+}\left(k, \tau_{1}\right)\right|^{2}-\left|A_{-}\left(k, \tau_{1}\right)\right|^{2}=1$. If the mixing coefficients $A_{ \pm}\left(k, \tau_{1}\right)$ are redefined as $\bar{A}_{+}=N_{\nu} A_{+}$and $\bar{A}_{-}=N_{\nu}^{*} \widetilde{A}_{-}$the general expressions of equations (A.1) and (A.2) become

$$
\begin{aligned}
& f_{k}(\tau)=\frac{1}{\sqrt{2 k}} \sqrt{k y}\left[\left(\bar{A}_{+}+\bar{A}_{-}\right) J_{\nu}(k y)+\mathrm{i}\left(\bar{A}_{-}-\bar{A}_{+}\right) Y_{\nu}(k y)\right], \\
& g_{k}(\tau)=\sqrt{\frac{k}{2}} \sqrt{k y}\left[\left(\bar{A}_{+}+\bar{A}_{-}\right) J_{\nu-1}(k y)+\mathrm{i}\left(\bar{A}_{-}-\bar{A}_{+}\right) Y_{\nu-1}(k y)\right] .
\end{aligned}
$$

Equations (A.6) and (A.7) can now be referred to the electric and magnetic mode functions of equations (3.1)-(3.3) evaluated at the end of inflation i.e.

$$
\begin{array}{ll}
\bar{f}_{k}=\frac{N_{\mu}}{\sqrt{2 k}} \sqrt{x_{1}} H_{\mu}^{(1)}\left(x_{1}\right), & \bar{g}_{k}=\sqrt{\frac{k}{2}} N_{\mu} \sqrt{x_{1}} H_{\mu+1}^{(1)}\left(x_{1}\right), \quad \gamma>1 / 2, \\
\bar{f}_{k}=\frac{N_{\mu}}{\sqrt{2 k}} \sqrt{x_{1}} H_{\mu}^{(1)}\left(x_{1}\right), & \bar{g}_{k}=-\sqrt{\frac{k}{2}} N_{\mu} \sqrt{x_{1}} H_{\mu-1}^{(1)}\left(x_{1}\right), \quad 0<\gamma<1 / 2,
\end{array}
$$

where, as in the bulk on the paper, $\bar{f}_{k}=f_{k}\left(-\tau_{1}\right)$ and $\bar{g}_{k}=g_{k}\left(-\tau_{1}\right)$. Since $x_{1}=k \tau_{1}<1$ for all the modes of the spectrum the ratio of the mode functions of equation (A.8) can always be evaluated in the small argument limit and it is:

$$
\left|\frac{k \bar{f}_{k}}{\bar{g}_{k}}\right|=\left|\frac{H_{|\gamma-1 / 2|}^{(1)}\left(x_{1}\right)}{H_{\gamma+1 / 2}^{(1)}\left(x_{1}\right)}\right| \rightarrow \frac{\Gamma(|\gamma-1 / 2|)}{\Gamma(\gamma+1 / 2)}\left(\frac{x_{1}}{2}\right)^{\gamma+1 / 2-|\gamma-1 / 2|} .
$$

The values of $f_{k}(\tau)$ and $g_{k}(\tau)$ for $\tau \geqslant-\tau_{1}$ will then be expressible in terms of $\bar{f}_{k}$ and $\bar{g}_{k}$ in the following manner

$$
\left(\begin{array}{c}
f_{k}(\tau) \\
g_{k}(\tau) / k
\end{array}\right)=\left(\begin{array}{ll}
A_{f f}\left(k, \tau, \tau_{1}\right) & A_{f g}\left(k, \tau, \tau_{1}\right) \\
A_{g f}\left(k, \tau, \tau_{1}\right) & A_{g g}\left(k, \tau, \tau_{1}\right)
\end{array}\right)\left(\begin{array}{c}
\bar{f}_{k} \\
\bar{g}_{k} / k
\end{array}\right) .
$$

The entries of the matrix appearing at the right-hand side of equation (A.10) have been given in equation (4.2); they follow directly from equations (A.6) and (A.7) once the various coefficients are made explicit in terms of equations (A.4) and (A.5).

\section{Appendix B. Transition matrix and decreasing gauge coupling}

When the gauge coupling decreases equations (2.41) and (2.42) describe the evolution of $\sqrt{\lambda}$ and of $\sqrt{\lambda}^{\prime}$ interpolating between the inflationary stage and the subsequent radiation epoch (see 
also figure 2). In full analogy with equations (A.1) and (A.2) the mode functions for $\tau \geqslant-\tau_{1}$ are determined in terms of a set of new coefficients $\widetilde{A}_{ \pm}\left(k, \tau_{1}\right)$ :

$$
\begin{aligned}
f_{k}(\tau) & =\frac{\sqrt{k y}}{\sqrt{2 k}}\left[\widetilde{A}_{-}\left(k, \tau_{1}\right) N_{\widetilde{\nu}} H_{\widetilde{\nu}}^{(1)}(k y)+\widetilde{A}_{+}\left(k, \tau_{1}\right) N_{\widetilde{\nu}}^{*} H_{\widetilde{\nu}}^{(2)}(k y)\right], \\
g_{k}(\tau) & =-\sqrt{\frac{k}{2}} \sqrt{k y}\left[\widetilde{A}_{-}\left(k, \tau_{1}\right) N_{\widetilde{\nu}} H_{\widetilde{\nu}+1}^{(1)}(k y)+\widetilde{A}_{+}\left(k, \tau_{1}\right) N_{\widetilde{\nu}}^{*} H_{\widetilde{\nu}+1}^{(2)}(k y)\right], \widetilde{\delta}>\frac{1}{2}, \\
g_{k}(\tau) & =\sqrt{\frac{k}{2}} \sqrt{k y}\left[\widetilde{A}_{-}\left(k, \tau_{1}\right) N_{\widetilde{\nu}} H_{\widetilde{\nu}-1}^{(1)}(k y)+\widetilde{A}_{+}\left(k, \tau_{1}\right) N_{\widetilde{\nu}}^{*} H_{\widetilde{\nu}-1}^{(2)}(k y)\right], 0<\widetilde{\delta}<1 / 2,
\end{aligned}
$$

where, in analogy with equation (A.3), the following auxiliary quantities have been introduced:

$$
\begin{aligned}
N_{\widetilde{\nu}} & =\sqrt{\frac{\pi}{2}} \mathrm{e}^{\mathrm{i} \pi(2 \widetilde{\nu}+1) / 4}, \quad y(\tau)=\tau+\tau_{1}(1+q), \\
q & =q(\widetilde{\delta}, \widetilde{\gamma})=\frac{\widetilde{\delta}}{\widetilde{\gamma}}, \quad \widetilde{\nu}=\left|\widetilde{\delta}-\frac{1}{2}\right| .
\end{aligned}
$$

The expression of $\widetilde{\nu}$ now depends on the range of $\widetilde{\delta}$. More precisely for $\widetilde{\delta}>1 / 2$ the mixing coefficients are:

$$
\begin{aligned}
& \widetilde{A}_{-}\left(k, \tau_{1}\right)=-\frac{\mathrm{i} \pi N_{\widetilde{\mu}}}{4 N_{\widetilde{\nu}}} \sqrt{q} x_{1}\left[H_{\widetilde{\mu}}^{(1)}\left(x_{1}\right) H_{\widetilde{\nu}+1}^{(2)}\left(q x_{1}\right)-H_{\widetilde{\mu}-1}^{(1)}\left(x_{1}\right) H_{\widetilde{\nu}}^{(2)}\left(q x_{1}\right)\right], \\
& \widetilde{A}_{+}\left(k, \tau_{1}\right)=-\frac{\mathrm{i} \pi N_{\widetilde{\mu}}}{4 N_{\widetilde{\nu}}^{*}} \sqrt{q} x_{1}\left[H_{\widetilde{\mu}-1}^{(1)}\left(x_{1}\right) H_{\widetilde{\nu}}^{(1)}\left(q x_{1}\right)-H_{\widetilde{\mu}}^{(1)}\left(x_{1}\right) H_{\widetilde{\nu}+1}^{(1)}\left(q x_{1}\right)\right], \widetilde{\delta}>1 / 2,
\end{aligned}
$$

where, as in equations (A.4) and (A.5), $x_{1}=k \tau_{1}$. In the range $0<\widetilde{\delta}<1 / 2$ we have instead that $\widetilde{A}_{ \pm}\left(k, \tau_{1}\right)$ are:

$$
\begin{aligned}
& \widetilde{A}_{-}\left(k, \tau_{1}\right)=\frac{\mathrm{i} \pi N_{\widetilde{\mu}}}{4 N_{\widetilde{\nu}}} \sqrt{q} x_{1}\left[H_{\widetilde{\mu}}^{(1)}\left(x_{1}\right) H_{\widetilde{\nu}-1}^{(2)}\left(q x_{1}\right)+H_{\widetilde{\mu}-1}^{(1)}\left(x_{1}\right) H_{\widetilde{\nu}}^{(2)}\left(q x_{1}\right)\right], \\
& \widetilde{A}_{+}\left(k, \tau_{1}\right)=-\frac{\mathrm{i} \pi N_{\widetilde{\mu}}}{4 N_{\widetilde{\nu}}^{*}} \sqrt{q} x_{1}\left[H_{\widetilde{\mu}-1}^{(1)}\left(x_{1}\right) H_{\widetilde{\nu}}^{(1)}\left(q x_{1}\right)+H_{\widetilde{\mu}}^{(1)}\left(x_{1}\right) H_{\widetilde{\nu}-1}^{(1)}\left(q x_{1}\right)\right], 0 \leqslant \widetilde{\delta}<1 / 2 .
\end{aligned}
$$

As in the case of the results of appendix A, because of the Wronskian normalization of equation (2.21), the mixing coefficients of equations (B.5) and (B.6) must satisfy $\left|\widetilde{A}_{+}\left(k, \tau_{1}\right)\right|^{2}-$ $\left|\widetilde{A}_{-}\left(k, \tau_{1}\right)\right|^{2}=1$. We can therefore summarize the situation by saying that while $A_{ \pm}\left(k, \tau_{1}\right)$ of equations (A.4) and (A.5) depend upon the range of $\gamma$, the $\widetilde{A}_{ \pm}\left(k, \tau_{1}\right)$ of equations (B.5) and (B.6) depend on the range of $\widetilde{\delta}$. Inserting the expressions of the coefficients (B.5) and (B.6) back into equations (B.1)-(B.3) the values of $f_{k}(\tau)$ and $g_{k}(\tau)$ for different values of $\widetilde{\delta}$ can then be referred to the inflationary mode functions at $\tau=-\tau_{1}$ directly obtainable from equations (3.22) and (3.23):

$$
\bar{f}_{k}=\frac{N_{\widetilde{\mu}}}{\sqrt{2 k}} \sqrt{x_{1}} H_{\widetilde{\mu}}^{(1)}\left(x_{1}\right), \quad \bar{g}_{k}=-N_{\widetilde{\mu}} \sqrt{\frac{k}{2}} \sqrt{x_{1}} H_{\widetilde{\mu}-1}^{(1)}\left(x_{1}\right),
$$

where $\widetilde{\mu}=\widetilde{\gamma}+1 / 2$. As in the case of equation (A.9) the ratio of the mode functions of equation (B.7) can always be evaluated in the small argument limit and the result is: 


$$
\left|\frac{k \bar{f}_{k}}{\bar{g}_{k}}\right|=\left|\frac{H_{\widetilde{\gamma}+1 / 2}^{(1)}\left(x_{1}\right)}{H_{|\widetilde{\gamma}-1 / 2|}^{(1)}\left(x_{1}\right)}\right| \rightarrow \frac{\Gamma(\widetilde{\gamma}+1 / 2)}{\Gamma(|\widetilde{\gamma}-1 / 2|)}\left(\frac{x_{1}}{2}\right)^{|\widetilde{\gamma}-1 / 2|-\widetilde{\gamma}-1 / 2} .
$$

The magnetic and the electric mode functions for $\tau>-\tau_{1}$ can therefore be related to $\bar{f}_{k}$ and $\bar{g}_{k}$ of equation (B.7) as:

$$
\left(\begin{array}{c}
f_{k}(\tau) \\
g_{k}(\tau) / k
\end{array}\right)=\left(\begin{array}{ll}
\widetilde{A}_{f f}\left(k, \tau, \tau_{1}\right) & \widetilde{A}_{f g}\left(k, \tau, \tau_{1}\right) \\
\widetilde{A}_{g f}\left(k, \tau, \tau_{1}\right) & \widetilde{A}_{g g}\left(k, \tau, \tau_{1}\right)
\end{array}\right)\left(\begin{array}{c}
\bar{f}_{k} \\
\bar{g}_{k} / k
\end{array}\right),
$$

where the explicit form of the various coefficients appearing at the right-hand side of equation (B.9) has been reported in equation (4.29) which is the analog ${ }^{26}$ of equation (4.2).

\section{Appendix C. Transition matrix and duality transformations}

The transition matrices of equations (A.10) and (4.29) are related by duality transformations. As it can be explicitly verified from equations (2.33), (2.34) and (2.41), (2.42) a duality transformation implies that $(\gamma, \delta)$ are transformed into $(\widetilde{\gamma}, \widetilde{\delta})$ :

$$
\sqrt{\lambda} \rightarrow 1 / \sqrt{\lambda} \Rightarrow \gamma \rightarrow \widetilde{\gamma}, \quad \delta \rightarrow \widetilde{\delta}
$$

It has been shown (see equation (3.33) and discussion therein) that during inflation the transformation $\gamma \rightarrow \widetilde{\gamma}$ exchanges electric into magnetic power spectra and vice versa. We shall now demonstrate that after inflation the corresponding duality transformation exchanges the elements of the transition matrices (A.10) and (4.29). Let us first start by noting that when $\delta \rightarrow \widetilde{\delta}$ the corresponding Bessel indices $\nu$ and $\widetilde{\nu}$ are related as:

$$
\delta \rightarrow \widetilde{\delta} \Rightarrow \nu \rightarrow 1-\widetilde{\nu}
$$

Equation (C.2) is a consequence of the definitions of $\nu(\delta)=\delta+1 / 2$ and $\widetilde{\nu}(\widetilde{\delta})=\mid \widetilde{\delta}-$ $1 / 2 \mid$ given, respectively, in equations (4.4) and (4.30). By now applying the transformation equation (C.2) to the matrix elements of $\mathcal{M}\left(\delta, x_{1}, x\right)$ and $\widetilde{\mathcal{M}}\left(\widetilde{\delta}, x_{1}, x\right)$ the following transformations are easily deduced:

$$
\begin{aligned}
& A_{f f} \rightarrow \widetilde{A}_{g g}, \quad A_{g g} \rightarrow \widetilde{A}_{f f}, \\
& A_{f g} \rightarrow-\widetilde{A}_{g f}, \quad A_{g f} \rightarrow-\widetilde{A}_{f g} .
\end{aligned}
$$

Equations (C.3) and (C.4) follow from the explicit expression of each matrix element. Consider, for instance, $A_{f f}\left(\delta, x_{1}, x\right)$ which we write, for immediate convenience, in terms of Hankel functions of first and second kinds:

$$
A_{f f}\left(\delta, x_{1}, x\right)=\frac{\mathrm{i}}{2} \sqrt{q x_{1}} \sqrt{k y}\left[H_{\nu}^{(1)}(k y) H_{\nu-1}^{(2)}\left(q x_{1}\right)-H_{\nu-1}^{(1)}\left(q x_{1}\right) H_{\nu}^{(2)}(k y)\right] .
$$

\footnotetext{
${ }^{26}$ Equations (4.29) and (4.2) do not coincide since $\nu$ and $\widetilde{\nu}$ are different. Under duality, the elements of the transition matrices have a well defined transformation law given in equation (4.48) (see also appendix $\mathrm{C}$ hereunder). Along a similar perspective, unlike the case of equations (3.2) and (3.3), the expression of $g_{k}(\tau)$ is unique for all the range of $\widetilde{\gamma}>0$; this occurrence follows from the difference between $\mu$ and $\widetilde{\mu}$.
} 
By now performing the transformation $\gamma \rightarrow \widetilde{\gamma}$ and $\delta \rightarrow \widetilde{\delta}$ (i.e. $\nu \rightarrow 1-\widetilde{\nu}$ ) we obtain, from equation (C.5),

$$
A_{f f}\left(\widetilde{\delta}, x_{1}, x\right)=\frac{\mathrm{i}}{2} \sqrt{q x_{1}} \sqrt{k y}\left[H_{1-\widetilde{\nu}}^{(1)}(k y) H_{-\widetilde{\nu}}^{(2)}\left(q x_{1}\right)-H_{-\widetilde{\nu}}^{(1)}\left(q x_{1}\right) H_{1-\widetilde{\nu}}^{(2)}(k y)\right] .
$$

It is understood that while doing the transformation also the arguments of $q$ and $y$ will change according to equations (4.4) and (4.30):

$$
q(\delta, \gamma)=\delta / \gamma \rightarrow \widetilde{\delta} / \widetilde{\gamma}=q(\widetilde{\delta}, \widetilde{\gamma}),
$$

and similarly for $y$. We now recall that the Hankel functions of generic index $\mu$ and generic argument $z$ obey $[42,43]$

$$
H_{-\mu}^{(1)}(z)=\mathrm{e}^{\mathrm{i} \pi \mu} H_{\mu}^{(1)}(z), \quad H_{-\mu}^{(2)}(z)=\mathrm{e}^{-\mathrm{i} \pi \mu} H_{\mu}^{(2)}(z) .
$$

Inserting equation (C.8) into equation (C.6) we therefore obtain

$$
A_{f f}\left(\widetilde{\delta}, x_{1}, x\right)=-\frac{\mathrm{i}}{2} \sqrt{q x_{1}} \sqrt{k y}\left[H_{\widetilde{\nu}-1}^{(1)}(k y) H_{\widetilde{\nu}}^{(2)}\left(q x_{1}\right)-H_{\widetilde{\nu}}^{(1)}\left(q x_{1}\right) H_{\widetilde{\nu}-1}^{(2)}(k y)\right],
$$

which coincides with $\widetilde{A}_{g g}\left(\widetilde{\delta}, x_{1}, x\right)$, as anticipated in equation (C.3). With a similar procedure also the remaining transformation rules of equations (C.3) and (C.4) can be easily deduced.

\section{ORCID iDs}

Massimo Giovannini (i) https://orcid.org/0000-0001-6854-2306

\section{References}

[1] Lichnerowicz A 1994 Magnetohydrodynamics: Waves and Shock Waves in Curved Space-Time (Dordrecht: Kluwer)

[2] Deser S and Teitelboim C 1976 Phys. Rev. D 131592

[3] Deser S 1982 J. Phys. A: Math. Gen. 151053

[4] Parker L 1968 Phys. Rev. Lett. 21562

[5] Birrell N D and Davies P C W 1982 Quantum Fields in Curved Space (Cambridge: Cambridge University Press)

[6] Parker L and Toms D 2009 Quantum Field Theory in Curved Spacetime: Quantized Fields and Gravity (Cambridge: Cambridge University Press)

[7] Fermi E 1949 Phys. Rev. 751169

[8] Alfvén H 1949 Phys. Rev. 751732

Richtmyer R D and Teller E 1949 Phys. Rev. 751729

[9] Hoyle F 1958 Proc. of Solvay Conference 'La structure et l'evolution de l'Univers' (Brussels) ed R Stoop p 59

[10] Zeldovich Y B 1965 Sov. Phys. - JETP 21656

Zeldovich Y B 1965 Zh. Eksp. Teor. Fiz. 48986

[11] Thorne K S 1967 Astrophys. J. 14851

[12] Ryan M P and Shepley L C 1975 Homogeneous Relativistic Cosmologies (Princeton, NJ: Princeton University Press)

[13] Harrison E R 1967 Phys. Rev. Lett. 181011

[14] Harrison E R 1968 Phys. Rev. 1671170

[15] Giovannini M 2000 Phys. Rev. D 62123505

[16] Alfvén H and Fälthammer C-G 1963 Cosmical Electrodynamics 2nd edn (Oxford: Clarendon)

[17] Parker E N 1979 Cosmical Magnetic Fields (Oxford: Clarendon) 
[18] Zeldovich Y B, Ruzmaikin A A and Sokoloff D D 1983 Magnetic Fields in Astrophysics (London: Gordon and Breach)

[19] Enqvist K 1998 Int. J. Mod. Phys. D 07331

[20] Giovannini M 2004 Int. J. Mod. Phys. D 13391

Kandus A, Kunze K and Tsagas C 2010 arXiv:1007.3891

[21] Han J L 2017 Annu. Rev. Astron. Astrophys. 55111

[22] Weinberg S 2008 Cosmology (Oxford: Oxford University Press)

[23] Ratra B 1992 Astrophys. J. Lett. 391 L1

Gasperini M, Giovannini M and Veneziano G 1995 Phys. Rev. Lett. 753796

Giovannini M 2001 Phys. Rev. D 64061301

[24] Bamba K and Sasaki M 2007 J. Cosmol. Astropart. Phys. JCAP02(2007)030

Bamba K 2007 J. Cosmol. Astropart. Phys. JCAP10(2007)015

Giovannini M 2008 Phys. Lett. B 659661

[25] Bamba K 2007 Phys. Rev. D 75083516

Martin J and Yokoyama J 2008 J. Cosmol. Astropart. Phys. JCAP01(2008)025

Giovannini M 2008 Lect. Notes Phys. 737863

Demozzi V, Mukhanov V and Rubinstein H 2009 J. Cosmol. Astropart. Phys. JCAP08(2009)025

[26] Brown I A 2011 Astrophys. J. 73383

Fujita T and Mukohyama S 2012 J. Cosmol. Astropart. Phys. JCAP10(2012)034

Kahniashvili T, Brandenburg A, Campanelli L, Ratra B and Tevzadze A G 2012 Phys. Rev. D 86 103005

Ferreira R Z and Ganc J 2015 J. Cosmol. Astropart. Phys. JCAP04(2015)029

Campanelli L 2016 Phys. Rev. D 93063501

Tsagas C 2014 arXiv:1412.4806

Tsagas C 2015 arXiv:1508.06604

[27] Peccei R D and Quinn H R 1977 Phys. Rev. Lett. 381440

Peccei R D and Quinn H R 1977 Phys. Rev. D 161791

[28] Kim J E 1987 Phys. Rep. 1501

Cheng H-Y 1988 Phys. Rep. 1581

Raffelt G G 1990 Phys. Rep. 198

Raffelt G G 2008 Lect. Notes Phys. 74151

[29] Carroll S M, Field G B and Jackiw R 1990 Phys. Rev. D 411231 Garretson W D, Field G B and Carroll S M 1992 Phys. Rev. D 465346

[30] Field G and Carroll S 2000 Phys. Rev. D 62103008

Giovannini M 2000 Phys. Rev. D 61063502

Giovannini M 2000 Phys. Rev. D 61063004

Bamba K 2006 Phys. Rev. D 74123504

Bamba K, Geng C Q and Ho S H 2008 Phys. Lett. B 664154

[31] Campanelli L 2009 Int. J. Mod. Phys. D 181395

Campanelli L and Giannotti M 2005 Phys. Rev. D 72123001

Campanelli L and Giannotti M 2006 Phys. Rev. Lett. 96161302

Giovannini M 2013 Phys. Rev. D 88063536

Bamba K 2015 Phys. Rev. D 91043509

[32] Giovannini M and Shaposhnikov M E 1998 Phys. Rev. D 572186

Giovannini M and Shaposhnikov M E 1998 Phys. Rev. Lett. 8022

Giovannini M 2000 Phys. Rev. D 61063004

[33] Giovannini M 2000 Phys. Rev. D 61063502

Dvornikov M and Semikoz V B 2012 J. Cosmol. Astropart. Phys. JCAP02(2012)040

Alexander S, Marcianò A and Spergel D 2013 J. Cosmol. Astropart. Phys. JCAP04(2013)046

Barrie N D and Kobakhidze A 2014 J. High Energy Phys. JHEP09(2014)163

[34] Giovannini M 2015 Phys. Rev. D 92121301

Giovannini M 2016 Phys. Rev. D 93103518

[35] Kharzeev D E 2014 Prog. Part. Nucl. Phys. 75133

Giovannini M 2013 Phys. Rev. D 88063536

[36] Kharzeev D E, McLerran L D and Warringa H J 2008 Nucl. Phys. A 803227

Fukushima K, Kharzeev D and Warringa H 2008 Phys. Rev. D 78074033

Kharzeev D E 2010 Ann. Phys. 325205

Giovannini M 2016 Phys. Rev. D 94081301 
[37] Sakharov A D 1966 Sov. Phys. - JETP 22241

Sakharov A D 1965 Zh. Eksp. Teor. Fiz. 49345

[38] Peebles P J E and Yu J T 1970 Astrophys. J. 162815

[39] Sunyaev R A and Zeldovich Y B 1970 Astrophys. Space Sci. 73

[40] Naselsky P and Novikov I 1993 Astrophys. J. 41314

[41] Weinberg S 2003 Phys. Rev. D 67123504

[42] Erdelyi A, Magnus W, Oberhettinger F and Tricomi F R 1953 Higher Trascendental Functions (New York: McGraw-Hill)

[43] Abramowitz M and Stegun I A 1972 Handbook of Mathematical Functions (New York: Dover)

[44] Giovannini M 2010 J. Cosmol. Astropart. Phys. JCAP04(2010)003

Giovannini M 2012 Phys. Rev. D 85101301

[45] Giovannini M 2009 Class. Quantum Grav. 26045004

Giovannini M 2020 Prog. Part. Nucl. Phys. 112103774

[46] Ito A, Soda J and Yamaguchi M 2020 arXiv:2009.03611 [astro-ph.CO]

[47] Bamba K, Elizalde E, Odintsov S D and Paul T 2020 arXiv:2012.12742 [gr-qc]

[48] Kobayashi T and Afshordi N 2014 J. High Energy Phys. JHEP10(2014)166

Stahl C, Strobel E and Xue S S 2016 Phys. Rev. D 93025004

Sharma R and Singh S 2017 Phys. Rev. D 96025012

Bavarsad E, Kim S P, Stahl C and Xue S S 2018 Phys. Rev. D 97025017

[49] Giovannini M 2018 Phys. Rev. D 97 061301(R)

[50] Ade P A R et al (BICEP2 and Keck Array Collaborations) 2016 Phys. Rev. Lett. 116031302

[51] Akrami Y et al (Planck Collaboration) 2020 Astron. Astrophys. 641 A10

[52] Kofman L, Linde A and Starobinsky A A 1994 Phys. Rev. Lett. 733195

[53] Kofman L, Linde A and Starobinsky A A 1997 Phys. Rev. D 563258

[54] Abbott L F, Farhi E and Wise M B 1982 Phys. Lett. B 11729

[55] Giovannini M 2018 Class. Quantum Grav. 35084003 